\title{
Medición de la vulnerabilidad monetaria en el área latinoamericana bajo un enfoque de señales “móviles”
}

\author{
EVA Medina Moral ${ }^{\text {a }}$, DAVId SAlvador Perucha ${ }^{\text {b }}$ \\ a Universidad Autónoma de Madrid, Facultad de CC.EE. y EE., C/ Francisco Tomás y Valiente, 5, \\ 28029 Cantoblanco (Madrid),España.E-mail: eva.medina@uam.es \\ b Instituto Universitario de Predicción Económica LR Klein, Universidad Autónoma de Madrid, C/ \\ Francisco Tomás y Valiente, 5, 28029 Cantoblanco (Madrid), España. E-mail: \\ david.salvador@inv.uam.es
}

\begin{abstract}
RESUMEN
Anticipar las crisis financieras permite a las autoridades aplicar medidas con la suficiente antelación para evitar la crisis y, con ello, sus efectos. Desde el colapso del Sistema Monetario Europeo (1992-1993), diferentes autores han tratado de anticipar estos episodios generando Sistemas de Alerta Temprana. Entre las metodologías utilizadas destaca el enfoque de señales, ampliamente implementado por su fácil interpretación. El objetivo de este estudio es diseñar un Sistema de Alerta Temprana, basado en el enfoque de señales, que permita que el punto de corte utilizado para identificar una señal varíe bajo escenarios temporales alternativos. Esta estrategia mejora la calidad de la predicción, ya que incorpora en el análisis la influencia que, sobre la evolución de los indicadores, podrín tener las medidas que las autoridades monetarias aplican desde que la primera señal es emitida.
\end{abstract}

Palabras clave: Sistema de Alerta Temprana (SAT), Enfoque de señales, Volatilidad Cambiaria, Crisis Financieras, Latinoamérica.

\section{Measurement of Monetary Vulnerability in the Latin American Area using a "Changing" Signals Approach}

\begin{abstract}
The anticipation of financial crises allows the authorities to apply measures well in advance to avoid the crisis and, with it, their effects. Since the collapse of the European Monetary System (1992-1993), different authors have tried to anticipate these episodes by generating Early Warning Systems. Among the methodologies used, the signal approach, widely implemented for its easy interpretation, stands out. The aim of this study is to design an Early Warning System, based on the signal approach, that allows the cut-off point used to identify a signal to vary under alternative time scenarios. This strategy improves the quality of the prediction, since it incorporates in the analysis the influence that, on the evolution of the indicators, might have the measures that the monetary authorities apply since the first signal is emitted.
\end{abstract}

Keywords: Early warning system (EWS), Signal Approach, Exchange rate volatility, Financial crises, Latin America.

Clasificación JEL: F31, F47, G15

Artículo recibido en marzo de 2017 y aceptado en marzo de 2018

Artículo disponible en versión electrónica en la página www.revista-eea.net, ref. ə-36205

ISSN 1697-5731 (online) - ISSN 1133-3197 (print) 


\section{INTRODUCCIÓN}

Una crisis financiera hace referencia a la crisis económica que tiene como principal causa la crisis del sistema financiero, es decir, no tanto la economía productiva de bienes tangibles pertenecientes al sector agrario, industrial o servicios (bienes que, si bien pueden verse afectados o incluso ser la causa estructural, no constituyen el epicentro u origen de la crisis), sino fundamentalmente el sistema bancario, el sistema monetario o ambos.

Entre los efectos de las crisis financieras podemos encontrar un conjunto de caídas abruptas de los precios de los activos (tanto financieros como reales), quiebras de empresas (financieras y/o no financieras) y deflaciones o fuertes perturbaciones en el mercado de divisas. Por otro lado, las crisis financieras tienen también un fuerte impacto a nivel social, pudiéndose observar sus consecuencias tanto a corto como a largo plazo en fenómenos tales como el desempleo, la inflación, el aumento de los intereses y de los niveles de endeudamiento, la recesión en general, o incluso en casos más extremos, la miseria y la pobreza.

Desde 1998, el Fondo Monetario Internacional establece que se pueden distinguir tres tipos de crisis financieras: 1) crisis bancaria; 2) crisis de deuda externa; 3) y crisis cambiaria. Este trabajo se centra en el estudio de la anticipación de las crisis cambiarias. La selección de esta tipología de crisis se debe a que son precisamente éstas, las crisis cambiarias, las que en mayor medida y con más intensidad se han presentado en los últimos años en el área geográfica analizada (Latinoamérica).

Una crisis cambiaria es la crisis experimentada por un país cuando se devalúa o deprecia el valor de cambio de su moneda. En estas situaciones, las perspectivas de pérdida de valor de la moneda y, con ello, de poder adquisitivo, generan en los inversores expectativas negativas que conducen a ataques especulativos contra la moneda, los cuales terminan incrementando la intensidad y dimensión de la crisis financiera. Ante una crisis cambiaria, los gobiernos, para defender su moneda, se ven obligados a hacer uso de grandes cantidades de reservas internacionales que venden en los mercados cambiarios; o a elevar considerablemente las tasas de interés como medida para atraer capital y, con ello, incrementar el interés por su moneda.

En este contexto, los gobiernos están interesados en anticipar la llegada de la crisis para tratar de aplicar medidas que o la frenen o reduzcan sus efectos. Con este objetivo la literatura empírica recoge numerosas investigaciones que tratan de construir un Sistemas de Alerta Anticipada que, a partir de la evolución de un conjunto de indicadores, permita predecir cuanto de probable es que ocurra una crisis. La mayoría de estos estudios trabajan con muestras que incluyen a distintos países que han registrado shocks en diferentes momentos del tiempo, y el objetivo es tratar de identificar los indicadores que registran una evolución 
inusual antes de la llegada de la crisis. Las metodologías utilizadas para ello son dos: modelos econométricos y enfoque de señales. Si bien la construcción de modelos econométricos permite agregar la información de todos los indicadores para obtener una cuantificación conjunta sobre la probabilidad de que las crisis ocurran, en el enfoque de señales cada indicador es analizado de manera individual. En concreto, partiendo de la idea de que la evolución del indicador tiende a mostrar comportamientos inusuales en los periodos que preceden a una crisis, el indicador emitirá una señal de crisis cuando su valor supere un determinado umbral o punto de corte.

Independientemente del enfoque utilizado en la construcción del Sistema de Alerta Temprana, existe un cierto consenso en la literatura empírica en relación a la elevada heterogeneidad que caracteriza a las crisis, observándose diferencias tanto en la dimensión temporal como espacial. Así, las crisis son distintas tanto en relación a las causas que las originan, las cuales han ido variando en el tiempo (Medina y Vicéns, 2006), como en relación a la evolución de las mismas según las características económicas del país que las sufre, pudiéndose identificar diferencias a nivel regional (Edison, 2000; El-Shazly, 2011). En este trabajo tratamos de mostrar evidencia empírica en este sentido, para lo cual se analizan las crisis ocurridas en 7 países latinoamericanos, tratando de identificar si existen diferencias en la detección de las mismas por país. Para ello se realiza el análisis para muestras individuales de países. En este sentido, el enfoque predictivo que se basa en la construcción de modelos econométricos cuenta con la limitación del tamaño muestral, el cual debe ser elevado para que los resultados sean consistentes. Sin embargo, el empleo de una técnica no parámetrica, como el enfoque de señales, resuelve este problema. Si bien la metodología tradicional del enfoque de señales trabaja conjuntamente con la muestra de países analizados, la aplicación de la misma a nivel país exige un elevado esfuerzo computacional, que crece a medida que lo hace el número de países e indicadores analizados. El trabajo presentado trata de ser un ejercicio, extrapolable a otras muestras geográficas y temporales, para tratar de ver si existen diferencias entre los países que justifiquen ese mayor esfuerzo computacional en la construcción de los Sistemas de Alerta Anticipada.

Por otro lado, en los Sistemas de Alerta construidos bajo el enfoque de señales, el punto de corte a partir del cual el indicador emite una señal permanece fijo durante todo el horizonte o ventana temporal de predicción, lo que implica que este umbral es único independientemente del momento en que se haga la predicción. Sin embargo, este punto de corte podría variar según la proximidad o lejanía de la crisis. En este sentido, una vez que el indicador ha emitido una señal de crisis, su evolución a medida que se acerca el shock podría variar. Por un lado, es de esperar un cierto deterioro del indicador a medida que la crisis está más cerca. Sin embargo, el indicador podría también registrar una cierta mejora si se viese impactado positivamente por las medidas que los gobiernos 
ponen en marcha cuando la primera señal de crisis es emitida. En este segundo caso la señal de crisis desaparecería si el punto de corte permanece estático, ya que el indicador se habrá alejado del umbral que anticipaba la crisis, por lo que el gobierno relajaría su política al reducirse la probabilidad de presencia del fenómeno. Sin embargo, los datos recogidos en este estudio demuestran que, aunque los indicadores mejoran en el tiempo, en muchos casos la crisis termina ocurriendo, por lo que el indicador debería emitir una señal de que la llegada de la crisis aún es posible. Para ello, el punto de corte debe ajustarse a la nueva situación, siendo necesario que su valor se modifique respecto al identificado en el periodo anterior.

Así, si bien el indicador, tanto con la metodología del punto de corte estático como con la del punto de corte móvil, habría emitido una señal de crisis cuando ésta estaba lejos en el tiempo, el enfoque de punto de corte estático habrá dejado de emitir la señal a medida que la crisis estaba más cerca. Sin embargo, bajo la metodología del punto de corte móvil el indicador seguirá emitiendo la señal ya que, aunque su evolución ha mejorado, no lo ha hecho lo suficiente como para evitar la crisis. La identificación de puntos de corte móviles permitiría, por tanto, mejoras en el porcentaje de crisis perdidas o no predichas por los Sistemas de Alerta Temprana. Además, en el cálculo de los puntos de corte móvil también se prioriza la reducción del número de crisis perdidas. Es decir, si bien su cálculo se realiza siguiendo la metodología tradicional, basada en minimizar la ratio ruido-señal la cual, a su vez, trata de minimizar las falsas alarmas, en nuestro trabajo imponemos una serie de restricciones en el proceso de optimización. En este sentido, y en línea con Babecký et al. (2014), creemos que un criterio puramente estadístico, como la relación ruido-señal, puede no ser suficiente para la evaluación de los modelos de alerta temprana desde la perspectiva de su uso con fines preventivos, ya que no tiene en cuenta las preferencias de los responsables políticos en relación con las crisis perdidas versus falsas alarmas. La inclusión de restricciones en el proceso de optimización permitirá la identificación de los indicadores más adecuados para anticipar las crisis en los países analizados.

En resumen, en este trabajo se trata de aportar evidencia empírica sobre si la heterogeneidad de las crisis debería tenerse en cuenta en la construcción de los Sistemas de Alerta Temprana, lo que conduciría a hacer análisis pormenorizados por país. Además, los resultados, también permitirán identificar la conveniencia o no de que los puntos de corte, o umbrales a partir de los cuales un indicador emite una señal, deban ir variando en el tiempo según la crisis se aproxima. Por último, siguiendo la metodología tradicional para calcular los puntos de corte de minimización de la ratio ruido-señal, incorporamos al ejercicio de optimización una serie de restricciones para prestar mayor atención al objetivo de reducir las crisis perdidas, el cual podría tener mayor interés para los gobiernos que el de sólo reducir las falsas alarmas. 
El trabajo se estructura en los siguientes apartados: en primer lugar se realiza una revisión de la literatura sobre la construcción de Sistemas de Alerta Temprana para la anticipación de crisis financieras, la cual permite identificar, primero, las distintas alternativas que existen para definir las crisis cambiarias, y, segundo, los indicadores clave para la detección de las mismas; a continuación se describe la metodología utilizada en la investigación en cuanto a la muestra de análisis empleada, la definición de crisis utilizada, los indicadores seleccionados para anticipar la crisis, así como la técnica utilizada para la detección de los puntos de corte móviles; en el siguiente apartado se interpretan los resultados del análisis realizado. El estudio concluye con un resumen de las principales conclusiones obtenidas.

\section{REVISIÓN DE LA LITERATURA}

Desde el colapso del Sistema Monetario Europeo (SME), producido durante la segunda mitad de 1992 y la primera de 1993, varios estudios se han centrado en anticipar las crisis financieras usando, en la mayoría de los casos, un conjunto de datos multi-país. Estos estudios dividen la muestra entre periodos de tranquilidad y periodos de turbulencias, e identifican como indicadores que permiten anticipar una crisis aquellos que registran comportamientos atípicos en los periodos anteriores a la crisis.

En los trabajos pioneros, las metodologías empleadas se pueden clasificar en tres categorías diferentes. La primera metodología corresponde al empleo de regresiones Probit y Logit donde la variable dependiente es una variable dicotómica que mide la ocurrencia de la crisis (Medina y Vicéns, 2006). Estos modelos devuelven la probabilidad de crisis que tiene el país en un periodo determinado, por lo que aumentos o disminuciones de las variables explicativas de la regresión generarán una mayor o menor (dependiendo de la dirección del efecto de la variable) probabilidad de que dicha crisis ocurra. También siguiendo la metodología de la regresión, pero en este caso lineal, hay estudios que utilizan estimaciones mínimo cuadráticas para explicar la evolución de una variable continua que mide la vulnerabilidad cambiaria de un país. Este índice de vulnerabilidad se calcula como un promedio ponderado de las pérdidas de las reservas internacionales y la variación del tipo de cambio real durante el periodo de crisis. Por último, la metodología del enfoque de señales, ampliamente utilizada en las aplicaciones reales de organismos gubernamentales y bancos centrales, analiza los indicadores de forma individual (análisis univariante). Bajo esta metodología, el indicador emitirá una señal si se supera un determinado umbral o punto de corte. Estos puntos de corte se calculan a partir de la identificación del percentil que minimiza el porcentaje de falsas señales o señales emitidas por el indicador que no terminan en crisis. 
Los primeros estudios en esta línea de investigación son los de Eichengreen, Rose y Wyplosz (1994, 1995, 1996), quienes construyeron el denominado modelo ERW utilizando como metodología un análisis de regresión Probit binario. En su último estudio, los autores analizaron un panel de datos trimestral de 20 países industriales ${ }^{1}$ en el periodo que abarca desde 1959 a 1993, con un total de 2.800 observaciones. Estudios posteriores tratan de analizar no sólo la llegada de la crisis sino si el impacto de ésta es igual en todos los shocks financieros. En esta línea se encuentra el trabajo de Frankel y Rose (1996) quienes aplican la metodología Probit a un panel anual de datos de 105 países en el periodo comprendido entre 1971 y 1992. Otros trabajos han tratado de incorporar mejoras tanto en la muestra de análisis como en la selección de indicadores utilizados. Es el caso de Berg y Pattillo (1999a, 1999b y 1999c) quienes en una serie de estudios evalúan los modelos existentes y realizan el desarrollo de un Sistema de Alerta Temprana mejorado, utilizando la metodología Probit, para su uso en el Fondo Monetario Internacional. También tratando de incorporar mejoras, pero en este caso en la definición de crisis cambiaria, Jacobs et al. (2005) utilizan distintas definiciones de crisis que combinan a través de un análisis factorial para construir la variable a modelizar a través de una regresión Logit. La muestra de análisis incluye datos mensuales de seis países asiáticos para el periodo comprendido entre enero de 1970 y finales del año 2001.

Aplicando la metodología de la regresión lineal, Sachs et al. (1996) analizan la vulnerabilidad de 20 países emergentes durante los periodos Noviembre de 1994 - Abril de 1995 (crisis del tequila iniciada en México) y Mayo de 1997 -

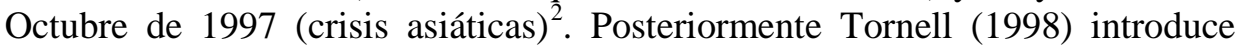
nuevas mejoras entre las que destaca la inclusión de tres países más en la muestra. Bussière y Mulder (1999) también introducen mejoras ampliando la muestra de análisis incluyendo la crisis de Rusia del 97 y 98.

Kaminsky, Lizondo y Reinhart (1998) son los primeros en utilizar el enfoque de señales en un Sistema de Alerta Temprana de crisis financieras. Los autores analizaron los datos mensuales de 15 países emergentes y 5 países industriales en el periodo que abarca desde 1970 a 1995. Posteriormente Kaminsky (1999) desarrolló un indicador compuesto, a partir del enfoque de señales, que tenía en cuenta las señales emitidas por todos los indicadores a la vez. Sin embargo se ha demostrado que el análisis conjunto de los indicadores ofrece peores resultados que el enfoque individual. Estudios más recientes como el de Edison (2000) y el

\footnotetext{
${ }^{1}$ Los países utilizados en la muestra en el modelo ERW son Estados Unidos, Reino Unido, Austria, Bélgica, Dinamarca, Francia, Italia, Países Bajos, Noruega, Suecia, Suiza, Canadá, Japón, Finlandia, Grecia, Irlanda, Portugal, España, Australia y Alemania.

${ }^{2}$ Los países utilizados en la muestra son Argentina, Brasil, Chile, Colombia, India, Indonesia, Jordania, Corea, Malasia, México, Pakistán, Perú, Filipinas, Sudáfrica, Sri Lanka, Taiwán, Tailandia, Turquía, Venezuela y Zimbabue.
} 
de Heun y Schlink (2004) mejoran el enfoque de señales desarrollado por Kaminsky, Lizondo y Reinhart, incluyendo nuevos países en las muestras de análisis así como nuevas variables.

Si bien estos estudios utilizan metodologías distintas, todos ellos comparten ciertos rasgos comunes en relación a los países analizados, a la definición de crisis y del horizonte temporal de predicción, y a la selección de indicadores. En concreto, en relación a la muestra de países, en los estudios se incluyen tanto países industriales como países en desarrollo, aunque predomina más el análisis de este último grupo.

En lo referente a la definición de los periodos de crisis, hay un amplio abanico de expresiones generadas por los diferentes autores. Todas ellas se inspiran en el modelo de presión del mercado cambiario de Girton y Roper (1977), quienes desarrollan un índice de presión del mercado cambiario combinando la variación del tipo de cambio con la variación en las reservas. Eichengreen, Rose, and Wyplosz (1996) argumentan que las tasas de interés pueden variar también como respuesta de los bancos centrales a los ataques especulativos, por lo que incorporan este indicador también en el cálculo del índice. Los autores definen un periodo de crisis cuando el índice de presión del mercado cambiario supera la media más un número de veces la desviación típica del índice (se prueba con 1 , 1,5 y 2 desviaciones típicas). Frankel y Rose (1996), argumentando que el aumento de las tasas de interés y las reservas internacionales no es una práctica habitual para hacer frente a un ataque especulativo en la mayoría de los países en desarrollo, utilizan sólo la variación del tipo de cambio nominal en su definición de crisis. Según esta definición se producirá una crisis si el aumento interanual del tipo de cambio nominal es superior al $25 \%$ y, a su vez, superior al $10 \%$ del aumento del tipo de cambio nominal del periodo anterior. Kamisnky, Lizondo y Reinhart (1998) utilizan el índice de presión del mercado cambiario pero excluyendo los diferenciales de tipos de interés, ya que advierten que las tasas de interés fueron controladas por los bancos centrales en su muestra. Los autores identifican una crisis cuando el valor del índice de presión del mercado cambiario, así calculado, supera la media más tres veces la desviación típica de los valores de dicho índice de la muestra analizada. Esta definición de crisis es adoptada también en el estudio de Heun y Schlink (2004) en su aplicación para Uganda. Zhang (2001) propone una versión alternativa del índice de presión cambiaria según la cual se descompone el índice en sus componentes (tipo de cambio, reservas y tipo de interés), de tal manera que se define una crisis cuando al menos se cumple una de las tres condiciones definidas como que cada componente por separado supere un determinado punto de corte. Otros autores construyen un índice de presión del mercado cambiario alternativo en el que sustituyen el tipo de interés, como tercer componente del índice de presión del mercado cambiario, por otros indicadores tales como el crédito doméstico (Pankki, 1999) o la base monetaria (Tanner, 2001). 
Una vez seleccionado el método para definir una crisis, es necesario identificar el horizonte o ventana temporal de predicción que determinará los valores de la variable objeto de estudio, ocurrencia de la crisis. Se entiende por ventana temporal de predicción el periodo para el cual se quiere anticipar la crisis, es decir, el tiempo que trascurre desde que se predice la crisis hasta que ésta ocurre realmente. Así, la variable de interés tomará el valor 1 el periodo en el que ocurre la crisis y los periodos anteriores incluidos en la ventana temporal de predicción, de modo que el resultado del análisis medirá la ocurrencia de la crisis en el horizonte temporal establecido. La elección de la ventana temporal de predicción debe buscar el equilibrio entre dos objetivos contrapuestos: por un lado, la garantía de acierto será superior cuanto menor sea el horizonte temporal de predicción, ya que los fundamentos económicos tienden a variar su evolución cuanto más cerca se encuentran de la crisis; por otro lado, lo deseable es que la crisis se anticipe lo antes posible para poder implementar las medidas necesarias que o bien la frenen o reduzcan sus efectos. Si bien algunos autores como Kaminsky et al. (1998) y Berg y Patillo (1999b) usan un horizonte temporal amplio, 24 meses, Bussière y Fratzscher (2006) defienden que una ventana de 12 meses es la mejor opción para encontrar el equilibrio entre los dos objetivos contrapuestos. Así, en su trabajo, realizan distintas pruebas para mostrar la robustez de sus resultados, y observan que para ventanas más amplias el número de falsas señales tiende a incrementarse. Otros autores, como Jacobs et al. (2005), prefieren también el uso de ventanas menos amplias para evitar que distintos shocks se mezclen en la construcción de la variable que identifica las crisis.

Por último, en relación a la selección de variables que anticipan las crisis, los estudios presentan un amplio y variado repertorio, muy vinculado a la muestra de países y periodo concreto analizado en cada caso (Medina y Vicéns, 2006; Haile y Pozo, 2008). La Tabla 1 recoge las variables utilizadas en distintos estudios $^{3}$ agrupadas en los siguientes bloques temáticos: indicadores del sector de economía real, sector exterior, sector público, así como indicadores financieros y de contexto global.

Los indicadores referentes al sector exterior y al sector financiero son los más utilizados en los distintos estudios, lo que hace prever una mayor capacidad predictiva de la economía financiera y del sector exterior para anticipar los shocks cambiarios. En concreto, en relación al sector exterior destaca el tipo de cambio real, exportaciones, importaciones y el indicador de términos de comercio como los más utilizados; mientras que en relación al sector financiero, son los agregados monetarios M1 y M2, el crédito interno, el tipo de interés real y los depósitos de los bancos comerciales los más frecuentes en los Sistemas de

\footnotetext{
${ }^{3}$ Los estudios fueron seleccionados por ser los trabajos pioneros a partir de los cuales se ha desarrollado la literatura empírica posterior.
} 
Alerta Temprana analizados. De los otros bloques temáticos, el índice de producción industrial, perteneciente al sector real, y los indicadores referentes al contexto global (tipo de interés de EEUU, diferencial entre el tipo de interés interno y externo y el crecimiento de las economías desarrolladas) también se utilizan con cierta frecuencia. Por último, merece la pena destacar que los indicadores del sector público son los menos utilizados, lo que anticipa el menor poder predictivo de estos indicadores para anticipar los shocks cambiarios.

Tabla 1

Selección de indicadores para anticipar una crisis cambiaria

\begin{tabular}{|c|c|c|c|c|c|c|c|c|c|}
\hline & ERW & FR & STVIT & KLR & BM & BBMP & EDISON & HS & JKL \\
\hline \multicolumn{10}{|l|}{ SECTOR REAL } \\
\hline Índice de Producción Industrial & $\mathrm{X}$ & & & $\mathrm{x}$ & & & $\mathrm{x}$ & $\mathrm{X}$ & \\
\hline PIB per cápita & & $x$ & & & & & & & $x$ \\
\hline Inflación & $\mathrm{X}$ & & & & & & & & \\
\hline Empleo & $\mathrm{X}$ & & & & & & & & \\
\hline Tasa de paro & $\mathrm{x}$ & & & & & & & & \\
\hline \multicolumn{10}{|l|}{ SECTOR EXTERIOR } \\
\hline Tipo de cambio real & & & $\mathrm{X}$ & $\mathrm{X}$ & $\mathrm{x}$ & $\mathrm{X}$ & $x$ & $\mathrm{X}$ & \\
\hline Exportaciones & & & & $\mathrm{x}$ & & $\mathrm{x}$ & $\mathrm{x}$ & $\mathrm{X}$ & $\mathrm{X}$ \\
\hline Importaciones & & & & $x$ & & & $x$ & $x$ & $x$ \\
\hline Saldo cuenta corriente (\% PIB) & $\mathrm{X}$ & $x$ & & & $\mathrm{x}$ & $\mathrm{x}$ & & & \\
\hline Indicador términos de comercio & & & & $\mathrm{x}$ & & $x$ & $\mathrm{X}$ & $x$ & $x$ \\
\hline Reservas (s/ Importaciones) & & $x$ & & & & & & & \\
\hline Flujo de inversión extranjera directa & & $x$ & & & & & & & \\
\hline Controles de capital & $\mathrm{x}$ & & & & & & & & \\
\hline \multicolumn{10}{|l|}{ SECTOR PÚBLICO } \\
\hline Saldo público (\% PIB) & $\mathrm{x}$ & $x$ & & & & & & & \\
\hline Ahorro interno & & & & & & & & & $x$ \\
\hline Deuda Pública & & $\mathrm{x}$ & & & & & $\mathrm{x}$ & & \\
\hline Ayudas para el desarrollo & & $\mathrm{x}$ & & & & & & & \\
\hline Victoria/derrota electoral de un gobierno & $\mathrm{X}$ & & & & & & & & \\
\hline \multicolumn{10}{|l|}{ INDICADORES FINANCIEROS } \\
\hline M1 & & & & $\mathrm{x}$ & & & $\mathrm{X}$ & $\mathrm{X}$ & $\mathrm{x}$ \\
\hline M2 & & & & $\mathrm{X}$ & & $\mathrm{X}$ & $\mathrm{X}$ & $\mathrm{X}$ & $\mathrm{X}$ \\
\hline Crédito interno & $\mathrm{x}$ & $\mathrm{X}$ & $\mathrm{x}$ & $\mathrm{x}$ & & & $\mathrm{X}$ & $\mathrm{X}$ & \\
\hline $\begin{array}{l}\text { Tipo de interés real (con respecto a los } \\
\text { depósitos) }\end{array}$ & & & & $\mathrm{x}$ & & & $\mathrm{x}$ & $\mathrm{x}$ & $\mathrm{x}$ \\
\hline Préstamos & & & & $x$ & & & $x$ & $x$ & \\
\hline Depósitos de bancos comerciales & & & & $x$ & & & $x$ & $\mathrm{X}$ & $\mathrm{x}$ \\
\hline Índice de precio de las acciones & & & & $x$ & & & $x$ & & \\
\hline \multicolumn{10}{|l|}{ CONTEXTO GLOBAL } \\
\hline Tipo de interés de EEUU & & $x$ & & & & & $x$ & & $x$ \\
\hline $\begin{array}{l}\text { Diferencial tipo de interés real interno y } \\
\text { externo }\end{array}$ & & & & $\mathrm{x}$ & & & $\mathrm{X}$ & $\mathrm{X}$ & \\
\hline "Output growth" de países desarrollados & & $\mathrm{X}$ & & & & & $x$ & & $\mathrm{X}$ \\
\hline Precio del petróleo & & & & & & & $\mathrm{x}$ & & \\
\hline
\end{tabular}

Nota: Los indicadores se miden en tasas de variación interanual para eliminar los efectos estacionales. Sólo en casos especiales los indicadores son medidos en desviaciones a la tendencia, como es el caso del tipo de cambio.

Fuente: Elaboración propia a partir de la información obtenida en ERW: Eichengreen, Rose y Wyplosz (1996); FR: Frankel y Rose (1996); STV/T: Sachs, Tornell y Velasco (1996) y Tornell (1998); KLR: Kaminsky, Lizondo y Reinhart (1998); BM: Bussière y Mulder (1999); BBMP: Berg, Borensztein, Milesi-Ferretti, y Pattillo (1999); EDISON: Edison (2000); HS: Heun y Schlink (2004); JKL: Jacobs, Kuper y Lestano (2005). 
En la última década, las turbulencias que ha registrado la economía a nivel mundial han despertado nuevamente el interés por el desarrollo de los Sistemas de Alerta Temprana como mecanismos para anticipar las crisis. En la literatura empírica más reciente existe un elevado número de estudios que, si bien se basan en los trabajos pioneros descritos anteriormente, buscan mejorar la capacidad predictiva de los mismos bajo distintas perspectivas. Algunos trabajos centran su atención en la construcción de la variable a modelizar. Así, Barberá et al. (2006) tratan de aportar evidencia empírica para la predicción directa del tipo de cambio; mientras que Angkinand et al. (2006) y Emin y Aytac (2016) analizan definiciones alternativas de crisis, modificando los componentes, ponderaciones y umbral del índice de presión del mercado cambiario, tratando de identificar cual aporta mayor capacidad predictiva al modelo. Otros trabajos, se focalizan en la selección de indicadores, como el estudio de Redondo y Rodríguez (2014) que trata de seleccionar los indicadores adecuados para el diseño de los Sistemas de Alerta Temprana aplicando la técnica del análisis discriminante.

Por último, la mayoría de los trabajos más recientes tienen como objetivo mejorar las técnicas estadísticas y econométricas empleadas en la predicción. En esta línea, algunos estudios incorporan variaciones de los modelos Logit y Probit para ajustar mejor las características específicas de las crisis. Así, Candelon et al. (2014) utilizan una versión dinámica de los modelos Logit, para incorporar la persistencia de la crisis al análisis, incluyendo entre los regresores retardos de la variable endógena; mientras que Caggaino et al. (2016) y Dawood et al. (2017) usan modelos Logit de respuesta multinomial que recogen no sólo la ocurrencia de la crisis sino también la duración e intensidad de la misma. Otros estudios usan métodos alternativos a los modelos Probit y Logit para construir el Sistema de Alerta Temprana. Es el caso de Rose y Spigel (2011) quienes utilizan modelos de ecuaciones estructurales para entender las causas de la crisis financiera de 2008 y poder anticipar situaciones similares en el futuro; Sevim et al. (2014) construyen el Sistema de Alerta Temprana a través del empleo de redes neuronales y árboles de decisión; mientras que Babecký et al. (2014) emplean la estadística bayesiana para la identificación de los indicadores que mejor anticipan la crisis.

La construcción de Sistemas de Alerta Temprana bajo el enfoque de señales se ha utilizado, en los estudios más recientes, como complemento a los resultados obtenidos con las técnicas estadísticas y econométricas. Así, en Bhattacharya et al. (2012) y Babecký et al. (2014) el enfoque de señales se aplica sólo a los indicadores que los modelos econométricos identificaron con mayor capacidad para anticipar las crisis. En este sentido, si bien las técnicas estadísticas y econométricas aportan información sobre los indicadores que mejor funcionan en la predicción de crisis, la facilidad interpretativa del enfoque de señales incrementa su valor para uso con fines prácticos (El-Shazly, 2011), ya que aportan más información para la toma de decisiones de acciones preventivas que 
permiten actuar sobre las causas de las crisis e inducir cambios en la probabilidad de ocurrencia de las mismas.

Es precisamente su uso con fines prácticos lo que requiere prestar más atención a la identificación del valor del umbral, o punto de corte asociado a la ocurrencia de la crisis, para tratar de evitar tanto las falsas alarmas como las crisis perdidas. Este estudio aporta evidencia sobre si los indicadores modifican su evolución según se aproxima la crisis, lo que debería tenerse en cuenta en la identificación de los puntos de corte. En este sentido, si los indicadores respondieran positivamente a las medidas preventivas que los gobiernos aplican cuando las señales de crisis son emitidas, el punto de corte debería ajustarse a la evolución que el indicador tiene a medida que la crisis está más cerca. Así, frente a la versión tradicional del enfoque de señales, según la cual el punto de corte es invariante durante todo el horizonte temporal de predicción, el uso del punto de corte móvil podría reducir el número de crisis no predichas por los Sistemas de Alerta Temprana en los casos en los que las medidas preventivas, si bien podrían inducir a una mejora en la evolución del indicador, no fueran suficientes para frenar la crisis. Además, el trabajo también trata de aportar evidencia empírica sobre si la heterogeneidad de las crisis podría generar una menor capacidad predictiva de los Sistemas de Alerta, siendo en ese caso más recomendable el estudio de muestras separadas de país.

\section{METODOLOGÍA EMPLEADA EN EL ANÁLISIS}

A continuación se describe la metodología empleada en este estudio, la cual, partiendo del trabajo pionero de Kaminsky et al. (1998), permite construir un Sistema de Alerta Temprana de predicción de crisis cambiarias en el área latinoamericana bajo un enfoque de señales, que trata de mejorar la capacidad predictiva del enfoque tradicional en relación al número de crisis perdidas o no predichas por el modelo. En concreto, frente a la identificación de un único punto de corte para el horizonte de predicción, tal y como se realiza en el enfoque de señales tradicional, este estudio pretende analizar si en distintos momentos temporales dicho punto de corte es el mismo o si, por el contrario, va variando a medida que se aproxima la crisis.

Así, si bien la base del Sistema de Alerta se apoya en que la crisis se detecta cuando el indicador registra una evolución inusual, no se tiene en cuenta que dicha evolución puede no ser la misma según el número de periodos que queden para la llegada de la crisis. De esta manera algo que se puede considerar inusual en un periodo concreto puede no serlo en periodos anteriores o viceversa. Para que esta situación pueda ser incorporada al Sistema de Alerta es necesario que el punto de corte vaya variando en el tiempo, lo que permitirá anticipar con más precisión las crisis según la lejanía o proximidad de las mismas. Además, también es importante tener en cuenta que las medidas aplicadas por los gobiernos, cuando 
una señal de crisis es emitida, podrían remitir la evolución del indicador falseando la apariencia de un comportamiento típico que no es capaz de detectar la llegada de la crisis, aun cuando ésta sí termina ocurriendo, quizá porque las medidas aplicadas no fueron suficientes como para frenarla. En esta situación, si el punto de corte permanece estático, el indicador dejará de emitir una señal de crisis cuando ésta está cerca. Sin embargo, si el punto de corte varía en el tiempo, su umbral se podrá ajustar a la evolución del indicador. Por tanto, el uso de puntos de corte móviles mejoraría la reducción de crisis perdidas o no predichas por el Sistema de Alerta Temprana, tanto cuando éstas están aún lejos en el tiempo, momento en que el indicador aún no ha iniciado su fase de mayor deterioro, como cuando están más próximas, momento en que el indicador podría, o no, revertir su tendencia.

Por otro lado, el trabajo presentado también pretende analizar si la heterogeneidad de las crisis podría estar reduciendo la capacidad predictiva de los modelos. En este sentido, el trabajar con muestras multi-país, a partir de las cuales se identifican patrones comunes a todos los países, puede reducir la capacidad de ajuste cuando las crisis son muy distintas entre ellas. En estos casos sería mejor realizar un análisis pormenorizado por país, donde al eliminar la heterogeneidad transversal, los puntos de corte se ajustarían mejor a las características económicas del país analizado siendo previsible una mejora en términos predictivos.

\subsection{Muestra de análisis}

La muestra utilizada en el estudio incluye datos mensuales para el periodo comprendido entre enero de 1990 y febrero de 2016 de siete países de América Latina: Argentina, Brasil, Chile, Colombia, México, Perú, y Venezuela. El análisis se centra en dicha área geográfica debido a que en los últimos 30 años ha experimentado diferentes crisis monetarias y representa un foco de vulnerabilidad cambiaria en el ámbito internacional. Además, esta localización geográfica permite dar respuesta a una necesidad del Centro de Estudios Latinoamericanos (CESLA), dedicado al análisis y seguimiento de las principales economías latinoamericanas, institución que ha financiado el estudio. Sin embargo, el ejercicio de análisis es fácilmente replicable a cualquier otra región de interés.

Los datos utilizados en el estudio tienen una periodicidad mensual y se han extraído de las estadísticas financieras internacionales del Fondo Monetario Internacional (FMI), de las estadísticas de la Comisión Económica para América Latina y el Caribe (CEPAL) y de la base de datos del Centro de Estudios Latinoamericanos (CESLA).

\subsection{Definición de crisis}

Para definir la ocurrencia de una crisis monetaria se utiliza el índice de presión 
del mercado cambiario desarrollado por Girton y Roper (1977), el cual se construye utilizando en su cálculo la variación intermensual del tipo de cambio y la variación intermensual ponderada de las reservas internacionales. Dicha ponderación se calcula como el cociente entre las desviaciones típicas de la variación del tipo de cambio y de las reservas internacionales. Si bien algunos autores añaden al cálculo del índice el tipo de interés (Eichengreen et al., 1996), en esta investigación no se ha incluido este indicador siguiendo la metodología empleada en Sachs et al. (1996), Kaminsky et al. (1998), Berg and Patillo (1999b), Edison (2000) y Heun y Schlink (2004) entre otros. Además, la especificidad de la muestra analizada, países del área latinoamericana, justifica también la no inclusión del tipo de interés en la definición de las crisis cambiarias. En este sentido, Angkinand et al. (2006), arrojan evidencia sobre la conveniencia de usar una definición de crisis específica dependiendo de las características de las crisis analizadas. En su trabajo valoran la capacidad del índice de presión del mercado cambiario para identificar crisis cambiarias utilizando diferentes componentes, ponderaciones y umbrales en la construcción del mismo. En el caso de los países latinoamericanos concluyen que los tipos de interés no funcionan bien en la identificación de las crisis ya que las autoridades monetarias de estos países se protegen de los ataques especulativos contra sus monedas a través de la venta de reservas en los mercados cambiarios, pero no utilizan como instrumento de defensa variaciones del tipo de interés. En concreto, sus resultados demuestran que el uso del tipo de interés en el cálculo del índice de presión especulativa incorpora ruido en la identificación de crisis.

La expresión matemática del índice de presión del mercado cambiario (IPMC) utilizada se recoge a continuación,

$$
I P M C_{t}=\Delta e_{t}-\left(\frac{\sigma \Delta e}{\sigma \Delta \mathrm{R}}\right) * \Delta R_{t},
$$

donde $e$ representa el tipo de cambio, $R$ las reservas, $\sigma$ la desviación típica de la serie, $\Delta$ la tasa de variación mensual, y $t$ el periodo muestral. La expresión recoge la idea de que las depreciaciones del tipo de cambio, que en la muestra analizada suelen ser compensadas con disminuciones de las reservas internacionales como herramienta utilizada por los bancos centrales para frenar la depreciación de sus monedas, van a representar una mayor vulnerabilidad monetaria para el país. Por ello, un mayor valor del índice de presión del mercado cambiario va a reflejar una mayor vulnerabilidad monetaria del país analizado. Para transformar el valor del índice en la definición de crisis hay que establecer un punto de corte. En la literatura empírica, este punto de corte se calcula como el valor de la media $(\mu)$ al que se suma un número de veces específico $(\beta)$ la desviación típica $(\sigma)$ del IPMC muestral. Utilizando este criterio, se producirá una crisis monetaria en un periodo determinado si el valor del IPMC es superior a ese punto de corte. En el caso de 
no superar ese valor, la observación se asociará a un periodo de calma cambiaria, tal y como se refleja en la siguiente expresión,

$$
\text { Crisis }=\left\{\begin{array}{c}
1 \text { si IPMC det para cada } i>\beta * \sigma_{i}^{I P M C}+\mu_{i}^{I P M C} \\
0 \text { en el resto decasos }
\end{array}\right\} .
$$

Se probaron distintos valores de $\beta$ para definir el mejor punto de corte. Se aplicó el análisis de la varianza Anova (test t) a los resultados obtenidos, no obteniéndose diferencias estadísticamente significativas, para un 95\% de confianza, entre los valores definidos para $\beta=1$ y $\beta=2$, por lo que se optó por trabajar con el valor intermedio. Así, un periodo registrará una crisis cambiaria cuando su valor del IPMC supere el valor medio más 1,5 veces la desviación típica del conjunto de valores del IPMC de la muestra.

Las crisis identificadas siguiendo esta metodología se muestran en la Tabla 2. De entre las crisis monetarias detectadas en el periodo muestral destacan la crisis del Tequila de 1994 originada en México, la crisis asiática de 1997 desencadenada por la devaluación del Bath Tailandés, la crisis brasileña producida a finales de la década de los noventa, derivada de la crisis rusa, y la crisis financiera internacional de 2007-2008. Todas estas crisis han afectado en un mayor o menor grado a los diferentes países que componen la muestra, en parte por el efecto contagio que caracteriza a estos fenómenos, tal y como analizan Haile y Pozo (2008) y Caramazza et al. (2004).

Tabla 2

Identificación de crisis cambiarias (enero de 1990 - febrero de 2016)

\begin{tabular}{|c|c|c|c|c|c|c|c|c|}
\hline & & \multicolumn{7}{|c|}{ PAÍSES } \\
\hline & & Argentina & Brasil & Chile & Colombia & México & Perú & Venezuela \\
\hline \multirow{16}{*}{ 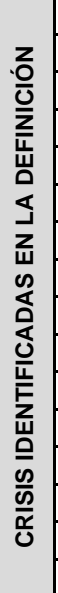 } & 1990 & $x$ & $x$ & & & & $x$ & $x$ \\
\hline & 1992 & & $\mathrm{x}$ & & $x$ & & & \\
\hline & 1993 & & $x$ & & $x$ & & & \\
\hline & Crisis del tequila de 1994 & $x$ & $x$ & $x$ & $x$ & $x$ & & $\mathrm{x}$ \\
\hline & 1995 & & & & & & & $x$ \\
\hline & Crisis asiática de 1997 & & $x$ & $\mathrm{x}$ & $\underline{x}$ & & $\mathrm{X}$ & \\
\hline & 1998 & & $x$ & & $x$ & & & \\
\hline & Corralito 2001 & $x$ & & $x$ & & & & $x$ \\
\hline & 2002 & & & & & & & $X$ \\
\hline & 2004 & & & & $x$ & & & \\
\hline & 2005 & $x$ & $x$ & $x$ & & & $x$ & \\
\hline & Crisis Internacional de 2007-08 & & $x$ & $x$ & $x$ & $\mathrm{x}$ & $x$ & $x$ \\
\hline & $2009-10$ & & & $x$ & & & & $x$ \\
\hline & 2012 & & & & & & & $x$ \\
\hline & 2013 & $x$ & & $X$ & & & & \\
\hline & 2015 & & & $x$ & & & & \\
\hline
\end{tabular}

Fuente: Elaboración propia. 


\subsection{Metodología para identificar el punto de corte “móvil”}

Tal y como ya se ha comentado, en la construcción del Sistema de Alerta Anticipada, para que la crisis sea predicha antes de que ésta ocurra, es necesario definir una ventana temporal de predicción, la cual incluye los periodos que trascurren desde que se predice la crisis hasta que ésta ocurre realmente. De esta manera, en la variable de interés una observación registrará una crisis en el momento en que ésta ocurre, siguiendo la definición descrita en el apartado anterior, así como en los periodos anteriores incluidos en la ventana temporal de predicción. Siguiendo a Jacobs et al. (2005) es preferible que la ventana no sea demasiado amplia para evitar que distintas crisis se fusionen en la construcción de la variable de interés, por lo que definimos una primera ventana temporal de 12 meses. Así, una observación registrará una crisis los 12 meses anteriores a que ésta ocurra.

Con la ventana temporal así definida, el indicador emitirá una señal de crisis en un periodo cuando el valor del indicador en ese periodo se sitúe por encima o por debajo de un umbral o punto de corte determinado. El punto de corte se corresponde con el percentil del conjunto de valores del indicador en la muestra que minimice la ratio ruido-señal. Esta ratio se obtiene a partir de una matriz de confusión (Tabla 3), y representa la relación entre la proporción de fallos condicionados a no haberse producido una crisis cambiaria y la proporción de aciertos condicionados a haberse producido la misma. En concreto, la expresión de la ratio es $[B /(B+D)] /[A /(A+C)]$, donde $A, B, C$ y $D$ se definen como:

- A: el indicador emite una señal condicionada y realmente se produce una crisis en dicho periodo.

- B: el indicador emite una señal condicionada pero no se produce realmente una crisis cambiaria en dicho periodo (falsos positivos o "mala señal”).

- C: el indicador no emite señal condicionada pero realmente sí se produce una crisis cambiaria (falsos negativos o "señal perdida").

- D: el indicador no emite señal condicionada de crisis y realmente no se produce una crisis cambiaria (verdaderos negativos o "buen silencio").

Tabla 3

Matriz de confusión

\begin{tabular}{|c|c|c|c|}
\cline { 3 - 4 } \multicolumn{2}{c|}{} & \multicolumn{2}{c|}{ Variable endógena (definición de crisis condicionada) } \\
\cline { 3 - 4 } Indicador & $\begin{array}{c}\text { Emite señal de crisis } \\
\text { condicionada }\end{array}$ & $\mathrm{A}$ & No se produce una crisis \\
\cline { 2 - 4 } & $\begin{array}{c}\text { No emite señal de crisis } \\
\text { condicionada }\end{array}$ & $\mathrm{C}$ & $\mathrm{B}$ \\
\hline
\end{tabular}

Fuente: Elaboración propia siguiendo el enfoque de señales original de Kaminsky, Lizondo y Reinhar (1998). 
Así definida la ratio ruido-señal, buscar su mínimo implica encontrar el percentil de la distribución del indicador con el que se reducen el número de falsas alarmas emitidas por el mismo. Con el objetivo de prestar atención también a la minimización de las crisis perdidas o crisis no predichas, cuyo interés de cara a la aplicación de medidas para frenar la crisis puede ser mayor que el de las falsas alarmas dados los costes tan elevados de que la crisis ocurra finalmente, se introducen las siguientes restricciones en el proceso de optimización matemático empleado:

- A > C, es decir, las señales ciertas deben ser igual o superiores a las crisis que el indicador no ha identificado.

- D > B, es decir, los periodos de calma cambiaria correctamente identificados deben ser igual o superiores a las falsas señales emitidas por el indicador.

- B > C, es decir, las falsas señales deben ser igual o superiores a las crisis no identificadas. Esta condición se ha establecido debido al mayor coste que representa una crisis no detectada y para la que no se han impuesto medidas hasta su llegada, en comparación con el coste en el que incurren las autoridades por implementar medidas para paliar una crisis que finalmente nunca se hubiese producido.

En el proceso de optimización se ha utilizado la herramienta Solver para un nivel de convergencia de 0,0001, una tasa de mutación de 0,075 y un valor de inicialización aleatorio de 0 . Esta herramienta permite localizar el percentil que cumple todos los requisitos y que será el utilizado para identificar las señales. En el enfoque tradicional de señales el percentil es el mismo para todos los países analizados ya que el proceso de optimización se realiza para la muestra total. Aunque el percentil sea el mismo para todos los países, el cálculo del umbral tiene en cuenta la distribución del indicador en cada país, de tal manera que el mismo percentil dará lugar a puntos de corte distintos en cada país. Sin embargo, la diversidad de crisis y la heterogeneidad existente entre países podría llevarnos a pensar que quizá el percentil a partir del cual se define el punto de corte debería ser también distinto en cada país. Para verificar esta hipótesis, en este estudio se presentan los resultados del cálculo del valor del percentil para cada indicador y en cada país. Si los percentiles identificados varían entre países, los resultados corroborarían una menor capacidad predictiva de los Sistemas de Alerta Anticipada cuando se trabaja con muestras multi-país, y que, por tanto, no tienen en cuenta las peculiaridades específicas de cada contexto económico.

Por último, dado que el objetivo del estudio es ver si el punto de corte se mantiene constante en el periodo de predicción o, por el contrario, varía a medida que se aproxima la crisis, es necesario definir distintas ventanas temporales de predicción, en cada una de las cuales será calculado un umbral distinto. En concreto, el punto de corte calculado, con la metodología descrita, para la ventana 
de predicción de 12 meses se compara con puntos de corte calculados en ventanas que anticipan la crisis cuando la proximidad de la misma es menor (9, 6 y 3 meses). En las nuevas ventanas de predicción un periodo registrará una crisis los 9, 6 y 3 meses anteriores, respectivamente, a que ésta se produzca. Para cada una de las ventanas se calculará el percentil que minimiza la ratio ruido-señal el cual, dado que la muestra ha sido segmentada por países, será distinto para cada indicador de cada país. Es decir, para cada uno de los indicadores seleccionados en cada uno de los países, el punto de corte tomará cuatro valores según el momento del tiempo en que la predicción sea hecha. Si se observa que los puntos de corte varían a medida que la crisis se aproxima, los resultados corroborarán la conveniencia de usar el sistema de puntos de corte móvil, que permite que el umbral que identifica la llegada de la crisis se pueda ir ajustando a medida que ésta se aproxima, evitando, con ello, el número de crisis no predichas.

Trabajar con este nivel de detalle exige un mayor esfuerzo computacional que el realizado aplicando la técnica más tradicional del enfoque de señales, según la cual el proceso de optimización arrojará como resultado el cálculo de un único percentil por indicador. La segmentación de la muestra por países y el cálculo de puntos de corte móvil implican que el proceso de optimización debe ser repetido varias veces, ya que es necesario obtener para cada indicador un número de percentiles que se calcula multiplicando el número de países por el número de ventanas de predicción que se quieren analizar. En concreto, y en nuestra muestra, en vez de un percentil por indicador habría que calcular 28 (7 países x 4 ventanas temporales de predicción). Los resultados obtenidos se presentan como un ejercicio para tratar de ver si la existencia de diferencias en los resultados justifica ese mayor esfuerzo computacional.

\subsection{Indicadores seleccionados para anticipar las crisis cambiarias}

Los indicadores utilizados han sido escogidos por ser los más frecuentes en la literatura empírica sobre Sistemas de Alerta Temprana para la detección de las crisis cambiarias (tal y como se ha detallado en el apartado de revisión de la literatura). En cualquier caso, el ejercicio que aquí se presenta trata de ejemplificar una metodología que puede ser extrapolable a otro conjunto de indicadores, países, e incluso ventanas temporales de predicción analizadas. En concreto se han utilizado los indicadores que se muestran en la Tabla 4. Esta tabla también recoge la dirección que causa la crisis en cada indicador. Es decir, el indicador emitirá una señal de crisis cuando su valor supere el percentil de punto de corte por encima, si el incremento del indicador se asocia a la llegada de la crisis, o por debajo, si el indicador tiende a deteriorarse a medida que la crisis se aproxima.

No se cuenta con información disponible de todas las series para todo el periodo temporal de análisis en todos los países, por lo que los indicadores que 
no disponen de esta información quedan excluidos del análisis. Las mediciones utilizadas en todos los casos son tasas de variación interanuales sobre los valores en niveles, excepto para el indicador tipo de interés (en cuyo caso se analizan las variaciones interanuales en niveles) y tipo de cambio real (en cuyo caso la tasa de variación se calcula para los valores medidos en desviaciones a la tendencia).

Tabla 4

Indicadores seleccionados en el Sistema de Alerta Temprana bajo el enfoque de señales "móviles"

\begin{tabular}{|l|c|l|}
\hline \multicolumn{1}{|c|}{ Indicador } & $\begin{array}{c}\text { Relación con la } \\
\text { ocurrencia de la crisis }\end{array}$ & $\begin{array}{c}\text { Valor del punto de corte que } \\
\text { causa la crisis }\end{array}$ \\
\hline Índice de producción industrial $^{(1)}$ & NEGATIVA & Crisis = valor $<$ punto de corte \\
\hline Índice de precios al consumidor & POSITIVA & Crisis $=$ valor $>$ punto de corte \\
\hline Exportaciones $(\$)$ & NEGATIVA & Crisis $=$ valor $<$ punto de corte \\
\hline Importaciones $(\$)$ & POSITIVA & Crisis $=$ valor $>$ punto de corte \\
\hline Saldo de la balanza comercial (\$) & NEGATIVA & Crisis $=$ valor $<$ punto de corte \\
\hline Términos de comercio & NEGATIVA & Crisis $=$ valor $<$ punto de corte \\
\hline Reservas Internacionales $(\$)$ & NEGATIVA & Crisis $=$ valor $<$ punto de corte \\
\hline Tipo de cambio real (moneda local/\$) ${ }^{(2)}$ & NEGATIVA & Crisis $=$ valor $<$ punto de corte \\
\hline Tipo de interés de los depósitos $(\%)$ & POSITIVA & Crisis $=$ valor $>$ punto de corte \\
\hline Depósitos a la vista (moneda local) & NEGATIVA & Crisis $=$ valor $<$ punto de corte \\
\hline Depósitos a plazo (moneda local) & NEGATIVA & Crisis $=$ valor $<$ punto de corte \\
\hline Agregado monetario M1 (moneda local) & POSITIVA & Crisis $=$ valor $>$ punto de corte \\
\hline Agregado monetario M2 (moneda local) & POSITIVA & Crisis $=$ valor $>$ punto de corte \\
\hline Tipo de interés de EEUU (\%) & POSITIVA & Crisis $=$ valor $>$ punto de corte \\
\hline
\end{tabular}

(1) En Perú y Colombia se utiliza como proxy el índice de producción manufacturera. (2) Los valores del tipo de cambio se expresan medidos en desviaciones a la tendencia.

Fuente: Elaboración propia.

\section{RESULTADOS DEL ANÁLISIS}

El proceso de optimización no se completó para todos los indicadores. Es decir, en algunos casos la convergencia no se logró debido a que el programa Solver no encontró una solución óptima. Estos son los indicadores en los que las restricciones impuestas, con el objetivo de reducir el número de crisis no predichas, no pudieron ser cumplidas. Consideramos, por tanto, que estos indicadores no son adecuados para predecir las crisis cambiarias en los países analizados, al no cumplir las condiciones establecidas para que los porcentajes de acierto sean óptimos. En concreto se trata de las importaciones, así como de aquellas variables que incluyen en su cálculo a las mismas (saldo de la balanza comercial y los términos de comercio). Quizá la debilidad de estos indicadores en la anticipación de crisis cambiaras se explica por tratarse de variables más asociados a "efecto" de la crisis que a "causa" de la misma, en el sentido de que una vez producida la depreciación, el encarecimiento de las importaciones 
podría provocar una fuerte caída en las mismas, lo que no tendría porque ocurrir antes de producirse el shock cambiario. Tampoco resultaron buenos indicadores para anticipar las crisis algunos de los indicadores financieros utilizados, como el agregado monetario M1 y los depósitos a plazo, aunque sí resultaron buenos predictores el agregado monetario M2 (que incluye el agregado M1 más los depósitos a plazo) y el indicador que mide los depósitos a la vista. En relación al tipo de interés de EEUU, la elevada incertidumbre que ha rodeado a este indicador en los últimos periodos de la muestra, así como la política restrictiva mantenida por la Reserva Federal (Vortelinos, 2016) durante un largo periodo de tiempo, invalidan su uso en el periodo muestral para anticipar las crisis cambiarias.

En cuanto a los indicadores que sí consiguen unos resultados adecuados y, por tanto, son buenos indicadores adelantados de las crisis latinoamericanas, éstos coinciden con los comúnmente identificados en la literatura empírica. Kaminsky et al. (1998) hacen una comparativa de los indicadores que mejores resultados obtienen para predecir crisis cambiarias en distintos estudios. Concluyen que las variables más útiles son reservas, tipo de cambio real, crecimiento del crédito, e inflación. Los resultados también suelen ser satisfactorios para las exportaciones, crecimiento del PIB y déficit fiscal. Los indicadores financieros funcionan bien sobre todo para anticipar crisis cambiarias que llevan asociadas crisis bancarias. Sin embargo, las variables de deuda externa, balanza por cuenta corriente y las relacionadas con el contexto global no suelen funcionar muy bien.

Resultados similares se obtienen en los trabajos presentados en la Tabla 1 de este estudio. Así, los indicadores que obtienen buenos resultados en mayor número de ocasiones son los relacionados con el sector exterior y, en concreto, el tipo de cambio, que resultó relevante en todos los trabajos que lo incluyeron en su análisis, y las exportaciones (Kaminsky et al. (1998), Berg et al. (1999) y Heun y Schlink (2004)). También, dentro de los indicadores de la economía real, el PIB y el índice de producción industrial (Frankel y Rose (1996); Kaminsky et al. (1998), Heun y Schlink (2004) y Jacobs et al. (2005)) suelen obtener buenos resultados para anticipar las crisis; y dentro de los financieros, el agregado monetario M2 es el que en mayor número de ocasiones resulta relevante (Kaminsky et al. (1998), Edison (2000), Heun y Schlink (2004) y Jacobs et al. (2005)). Estudios más recientes confirman estos resultados, los cuales también coinciden con los obtenidos en este trabajo, en el cual los indicadores con una buena capacidad para anticipar las crisis en los países analizados son: dentro de los relacionados con la economía real, el índice de producción industrial y los precios; de los incluidos en la categoría de sector exterior, las exportaciones, el tipo de cambio y las reservas; y de los indicadores financieros, el agregado monetario M2, los depósitos a la vista y el tipo de interés. 


\subsection{Heterogeneidad entre países}

A continuación se detallan los resultados obtenidos tras los cálculos realizados. En la Tabla 5 se recogen los percentiles, para cada indicador, que minimizan la ratio ruido-señal en cada uno de los 7 países de la muestra y para cada una de las ventanas temporales de predicción analizadas. La interpretación de los datos debe realizarse de la siguiente manera. El percentil 0,17 para el indicador de exportaciones en Argentina, indica que la señal será emitida, para una ventana temporal de 12 meses, cuando el valor de este indicador sea menor que el valor de la distribución de la serie (las exportaciones en Argentina) que deja por debajo de él el 17\% de las observaciones. Así mismo, el percentil 0,72 del indicador M2 en la ventana temporal de 12 meses de Argentina, indica que este indicador emitirá la señal cuando su valor se sitúe por encima del valor de la distribución de la serie que deja por debajo de él al 72\% de las observaciones. Es decir, los valores de los percentiles serán bajos en los indicadores en los que un deterioro del mismo anticipa la llegada de la crisis, mientras que se obtendrán percentiles altos para los indicadores donde una evolución creciente se asocie con la ocurrencia de la crisis.

Las últimas columnas de la tabla recogen los valores medios, así como la dispersión registrada entre países medida a través de la desviación típica y el coeficiente de variación. Además, se incluye un gráfico para cada uno de estos estadísticos, que recoge la evolución de los mismos en las distintas ventanas de predicción, con el objetivo de ver si los valores de los percentiles varían a medida que se acerca la crisis. Para identificar los indicadores donde la dispersión es más elevada, los valores del coeficiente de variación se han sombreado, de menos a más oscuro, según que la dispersión sea mayor o menor en base a los siguientes intervalos: menor de 0,3 ; entre 0,3 y 0,5; entre 0,5 y 0,7; mayor de 0,7.

Lo primero que se observa es que en varios de los indicadores los valores obtenidos difieren entre países. En concreto, los coeficientes de variación resultaron elevados para el índice de producción industrial, las exportaciones y las reservas, y, en menor medida, para la variable de depósitos a la vista y tipo de cambio. Para las variables M2, precios y tipo de interés los percentiles muestran valores más similares entre países.

En cuanto a la evolución de los valores medios se observa que el percentil medio tiende a crecer a medida que se acerca la crisis en los siguientes indicadores: tipo de cambio, exportaciones, depósitos a la vista e índice de producción industrial. Esta evolución indica que, dado que se trata de indicadores en los que un deterioro del mismo se asocia con la llegada de la crisis, el punto de corte debería ser menos exigente a medida que la crisis está cerca. Es decir, su evolución mejora antes de que la crisis se acerque, quizá porque se ven influenciados por las medidas correctivas aplicadas por los gobiernos para evitar que el shock cambiario ocurra. 


\section{Tabla 5}

Valor de los percentiles que minimizan la ratio ruido-señal

\begin{tabular}{|c|c|c|c|c|c|c|c|c|c|c|c|c|c|}
\hline & \multirow{2}{*}{ ARG } & \multirow{2}{*}{ BRA } & \multirow{2}{*}{$\mathrm{CHI}$} & \multirow{2}{*}{ COL } & \multirow{2}{*}{ MEX } & \multirow{2}{*}{ PER } & \multirow{2}{*}{ VEN } & \multicolumn{2}{|c|}{ PROMEDIO } & \multicolumn{2}{|c|}{ DESVIACIÓN TÍPICA } & \multicolumn{2}{|c|}{ COEF. VARIACIÓN } \\
\hline & & & & & & & & Valor & Evolución & Valor & Evolución & Valor & Evolución \\
\hline \multicolumn{14}{|c|}{ EXPORTACIONES: relación NEGATIVA con la ocurrencia de una crisis } \\
\hline $12 \mathrm{~m}$ & 0,17 & 0,15 & 0,12 & 0,08 & 0,09 & 0,07 & - & 0,11 & & 0,04 & & 0,34 & \\
\hline $9 m$ & 0,10 & 0,22 & 0,23 & 0,08 & 0,09 & 0,07 & 0,00 & 0,11 & & 0,08 & & 0,73 & \\
\hline $6 m$ & 0,10 & 0,24 & 0,19 & 0,11 & 0,08 & 0,07 & 0,16 & 0,14 & & 0,06 & & 0,45 & \\
\hline $3 m$ & 0,13 & 0,28 & 0,29 & 0,33 & 0,21 & 0,14 & 0,10 & 0,21 & & 0,09 & & 0,43 & \\
\hline \multicolumn{14}{|c|}{ RESERVAS: relación NEGATIVA con la ocurrencia de una crisis } \\
\hline $12 m$ & 0,14 & 0,11 & 0,21 & 0,13 & 0,06 & 0,21 & 0,10 & 0,14 & & 0,05 & & 0,39 & \\
\hline $9 m$ & 0,10 & 0,19 & 0,19 & 0,11 & 0,06 & 0,31 & 0,10 & 0,15 & & 0,08 & & 0,56 & \\
\hline $6 m$ & 0,07 & 0,24 & 0,21 & 0,15 & 0,06 & 0,20 & 0,16 & 0,16 & & 0,07 & & 0,44 & \\
\hline $3 m$ & 0,05 & 0,34 & 0,25 & 0,18 & 0,04 & 0,10 & 0,31 & 0,18 & & 0,12 & & 0,67 & \\
\hline \multicolumn{14}{|c|}{ TIPO DE CAMBIO REAL: relación NEGATIVA con la ocurrencia de una crisis } \\
\hline $12 \mathrm{~m}$ & 0,45 & 0,12 & 0,26 & 0,29 & 0,21 & 0,16 & 0,29 & 0,25 & & 0,11 & & 0,43 & \\
\hline $9 m$ & 0,27 & 0,29 & 0,27 & 0,33 & 0,20 & 0,15 & 0,27 & 0,25 & & 0,06 & & 0,24 & \\
\hline $6 m$ & 0,27 & 0,27 & 0,27 & 0,31 & 0,28 & 0,21 & 0,22 & 0,26 & & 0,04 & & 0,14 & \\
\hline $3 m$ & 0,30 & 0,41 & 0,27 & 0,46 & 0,37 & 0,45 & 0,21 & 0,35 & & 0,10 & & 0,27 & \\
\hline \multicolumn{14}{|c|}{ AGREGADO MONETARIO M2: relación POSITIVA con la ocurrencia de una crisis } \\
\hline $12 \mathrm{~m}$ & 0,72 & 0,71 & 0,68 & 0,73 & 0,68 & 0,80 & 0,36 & 0,67 & & 0,14 & & 0,21 & \\
\hline $9 m$ & 0,65 & 0,80 & 0,55 & 0,72 & 0,61 & 0,81 & 0,39 & 0,65 & & 0,15 & & 0,23 & \\
\hline $6 m$ & 0,60 & 0,80 & 0,56 & 0,64 & 0,68 & 0,81 & 0,86 & 0,71 & & 0,12 & & 0,16 & \\
\hline $3 m$ & 0,58 & 0,80 & 0,57 & 0,73 & 0,65 & 0,81 & 0,77 & 0,70 & & 0,10 & & 0,14 & \\
\hline \multicolumn{14}{|c|}{ DEPÓSITOS A LA VISTA: relación NEGATIVA con la ocurrencia de una crisis } \\
\hline $12 \mathrm{~m}$ & 0,24 & 0,21 & 0,17 & 0,16 & 0,06 & 0,11 & 0,24 & 0,17 & & 0,07 & & 0,39 & \\
\hline $9 m$ & 0,24 & 0,25 & 0,17 & 0,18 & 0,06 & 0,21 & 0,17 & 0,18 & & 0,06 & & 0,35 & \\
\hline $6 m$ & 0,32 & 0,25 & 0,26 & 0,18 & 0,06 & 0,21 & 0,16 & 0,21 & & 0,08 & & 0,40 & \\
\hline $3 m$ & 0,38 & 0,23 & 0,37 & 0,18 & 0,05 & 0,31 & 0,17 & 0,24 & & 0,12 & & 0,49 & \\
\hline \multicolumn{14}{|c|}{ TIPO DE INTERÉS: relación POSITIVA con la ocurrencia de una crisis } \\
\hline $12 m$ & 0,91 & 0,85 & 0,78 & 0,82 & 0,91 & 0,74 & 0,81 & 0,83 & & 0,06 & & 0,08 & \\
\hline $9 m$ & 0,92 & 0,90 & 0,79 & 0,89 & 0,83 & 0,74 & 0,79 & 0,84 & & 0,07 & & 0,08 & \\
\hline $6 m$ & 0,91 & 0,91 & 0,62 & 0,80 & 0,91 & 0,74 & 0,78 & 0,81 & & 0,11 & & 0,13 & \\
\hline $3 m$ & 0,93 & 0,92 & 0,62 & 0,75 & 0,90 & 0,70 & 0,80 & 0,80 & & 0,12 & & 0,15 & \\
\hline \multicolumn{14}{|c|}{ ÍNDICE DE PRECIOS AL CONSUMO: relación POSITIVA con la ocurrencia de una crisis } \\
\hline $12 \mathrm{~m}$ & 0,78 & 0,67 & 0,59 & 0,66 & 0,52 & 0,65 & 0,65 & 0,65 & & 0,08 & & 0,12 & \\
\hline $9 \mathrm{~m}$ & 0,81 & 0,69 & 0,59 & 0,75 & 0,52 & 0,65 & 0,66 & 0,67 & & 0,10 & & 0,15 & \\
\hline $6 m$ & 0,81 & 0,78 & 0,68 & 0,73 & 0,55 & 0,65 & 0,61 & 0,69 & & 0,09 & & 0,14 & \\
\hline $3 m$ & 0,81 & 0,85 & 0,64 & 0,73 & 0,53 & 0,65 & 0,63 & 0,69 & & 0,11 & & 0,16 & \\
\hline \multicolumn{14}{|c|}{ ÍNDICE DE PRODUCCIÓN INDUSTRIAL: relación NEGATIVA con la ocurrencia de una crisis } \\
\hline $12 \mathrm{~m}$ & 0,09 & 0,14 & 0,19 & 0,02 & 0,04 & 0,02 & - & 0,08 & & 0,07 & & 0,83 & \\
\hline $9 m$ & 0,24 & 0,15 & 0,18 & 0,02 & 0,07 & 0,14 & - & 0,13 & & 0,08 & & 0,61 & \\
\hline $6 m$ & 0,23 & 0,15 & 0,18 & 0,02 & 0,09 & 0,15 & - & 0,14 & & 0,07 & & 0,53 & \\
\hline $3 m$ & 0,23 & 0,14 & 0,24 & 0,02 & 0,15 & 0,32 & - & 0,18 & & 0,10 & & 0,57 & \\
\hline
\end{tabular}

Nota: Los cálculos se realizan para las 4 ventanas temporales de predicción incluidas en el análisis: 12 meses (12m); 9 meses $(9 \mathrm{~m}) ; 6$ meses $(6 \mathrm{~m})$ y 3 meses $(3 \mathrm{~m})$. Los valores del coeficiente de variación aparecen sombrados según su cuantía, siendo el sombreado más oscuro el que se corresponde con una mayor dispersión según la siguiente escala: menor de 0,3; entre 0,3 y 0,5; entre 0,5 y 0,7; mayor de 0,7.

Fuente: Elaboración propia. 
En relación a la evolución que registra la dispersión en las distintas ventanas temporales se observa que ésta tiende a aumentar en los indicadores de reservas, exportaciones, depósitos a la vista y tipo de interés, casos en los que el coeficiente de variación casi se duplica desde la ventana de predicción de 12 meses hasta la de 3 meses.

En resumen, los resultados confirman que en cinco de los ocho indicadores analizados sería aconsejable realizar los análisis para muestras individuales de países, ya que la elevada heterogeneidad registrada entre las series de cada país conduce a la identificación de percentiles distintos en cada indicador. Estos indicadores son el índice de producción industrial, las exportaciones, las reservas, los depósitos a la vista y el tipo de cambio. Además se observa que en todos estos indicadores, excepto en las reservas, el valor del percentil tiende a aumentar cuando la crisis está más cerca, lo que también se interpreta como un indicativo de la necesidad de identificar un punto de corte móvil en el tiempo. Esta idea será tratada con mayor profundidad en el siguiente apartado. Por último, en los indicadores de exportaciones y depósitos a la vista no sólo aumenta el valor del percentil cuando se aproxima la crisis sino que también lo hace el valor de su dispersión. La elevada heterogeneidad que registra la evolución de estos indicadores, tanto por países como en el tiempo, debería ser tenida en cuenta en la construcción de los Sistemas de Alerta Temprana con el objetivo de mejorar su capacidad predictiva, lo que exigiría su análisis a través de muestras por país en vez de muestras multi-país.

\subsection{Identificación de puntos de corte "móviles"}

En base a los percentiles identificados en la Tabla 5, es posible calcular el valor de la distribución, de cada indicador, que permitirá definir el umbral o punto de corte a partir del cual la señal de crisis será emitida. En la Tabla 6 se recogen dichos umbrales para los 8 indicadores, en cada uno de los 7 países de la muestra y para cada una de las 4 ventanas temporales de predicción analizadas. Dado que los datos se expresan en tasas de variación interanual (excepto para el tipo de interés cuya unidad de medida es la diferencia interanual de los datos en niveles) el dato de $-6 \%$ para el indicador de exportaciones en Argentina debe interpretarse como que la señal será emitida, en una ventana temporal de 12 meses, cuando la caída interanual de las exportaciones supere el valor de $-6 \%$. Esta misma interpretación debe aplicarse a todos los indicadores cuyo deterioro anuncian la llegada de la crisis (reservas, tipo de cambio, depósitos a la vista e índice de producción industrial). Sin embargo, en los casos en que la llegada de la crisis se asocia a una evolución creciente del indicador (M2, precios y tipo de interés) la interpretación debe realizarse en términos de superar el umbral. Es decir, el punto de corte de $32 \%$ para la variable M2 en Argentina debe interpretarse de la siguiente manera: cuando la variación interanual de esta 
variable supere dicho punto de corte el indicador emitirá una señal, ya que es probable que ocurra una crisis en los doce meses siguientes.

\section{Tabla 6}

Valor de los puntos de corte en cada ventana temporal de predicción

\begin{tabular}{|c|c|c|c|c|c|c|c|c|c|c|}
\hline & $A P C$ & PPA & $\mathrm{CH}$ & $\mathrm{CO}$ & AEY & PEP & VEN & \multicolumn{3}{|c|}{ EVOLUCIÓN DEL PUNTO DE CORTE } \\
\hline & ARG & BRA & CHI & COL & MEXX & PER & VEN & - exigente & + exigente & Constante \\
\hline \multicolumn{11}{|c|}{ EXPORTACIONES: relación NEGATIVA con la ocurrencia de una crisis } \\
\hline $12 m$ & $-6 \%$ & $-7 \%$ & $-10 \%$ & $-14 \%$ & $-5 \%$ & $-18 \%$ & - & \multirow{4}{*}{$\begin{array}{c}\text { Brasil, } \\
\text { Chile, } \\
\text { Colombia, } \\
\text { México, } \\
\text { Perú, } \\
\text { Venezuela }\end{array}$} & \multirow{4}{*}{ Argentina } & \\
\hline $9 \mathrm{~m}$ & $-13 \%$ & $-3 \%$ & $-5 \%$ & $-14 \%$ & $-5 \%$ & $-18 \%$ & $-54 \%$ & & & \\
\hline $6 \mathrm{~m}$ & $-13 \%$ & $-2 \%$ & $-6 \%$ & $-9 \%$ & $-5 \%$ & $-17 \%$ & $-23 \%$ & & & \\
\hline $3 m$ & $-10 \%$ & $-1 \%$ & $-1 \%$ & $2 \%$ & $3 \%$ & $-10 \%$ & $-29 \%$ & & & \\
\hline \multirow{2}{*}{\multicolumn{11}{|c|}{ RESERVAS: relación NEGATIVA con la ocurrencia de una crisis }} \\
\hline & & & & & & & & & & \\
\hline $12 \mathrm{~m}$ & $-19 \%$ & $-9 \%$ & $-1 \%$ & $-2 \%$ & $-13 \%$ & $-2 \%$ & $-32 \%$ & \multirow{5}{*}{$\begin{array}{c}\text { Brasil, } \\
\text { Colombia, } \\
\text { Venezuela }\end{array}$} & \multirow{5}{*}{$\begin{array}{l}\text { Argentina, } \\
\text { México, } \\
\text { Perú }\end{array}$} & \multirow{5}{*}{ Chile } \\
\hline $9 m$ & $-26 \%$ & $-3 \%$ & $-1 \%$ & $-3 \%$ & $-13 \%$ & $4 \%$ & $-32 \%$ & & & \\
\hline $6 m$ & $-30 \%$ & $0 \%$ & $-1 \%$ & $-1 \%$ & $-14 \%$ & $-2 \%$ & $-27 \%$ & & & \\
\hline $3 m$ & $-35 \%$ & $4 \%$ & $0 \%$ & $0 \%$ & $-28 \%$ & $-6 \%$ & $-15 \%$ & & & \\
\hline & 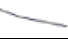 & $\longrightarrow$ & $\sim$ & 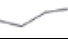 & & 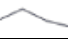 & _ & & & \\
\hline \multicolumn{11}{|c|}{ TIPO DE CAMBIO REAL: relación NEGATIVA con la ocurrencia de una crisis } \\
\hline $12 m$ & $-20 \%$ & $-119 \%$ & $-79 \%$ & $-40 \%$ & $-53 \%$ & $-84 \%$ & $-5 \%$ & \multirow{5}{*}{$\begin{array}{c}\text { Argentina, } \\
\text { Brasil, } \\
\text { Colombia, } \\
\text { México, } \\
\text { Perú }\end{array}$} & \multirow{5}{*}{ Venezuela } & \multirow{4}{*}{ Chile } \\
\hline $9 m$ & $-10 \%$ & $-49 \%$ & $-74 \%$ & $-35 \%$ & $-54 \%$ & $-89 \%$ & $-5 \%$ & & & \\
\hline $6 m$ & $-10 \%$ & $-51 \%$ & $-77 \%$ & $-39 \%$ & $-40 \%$ & $-67 \%$ & $-6 \%$ & & & \\
\hline $3 m$ & $-13 \%$ & $-27 \%$ & $-79 \%$ & $-13 \%$ & $-28 \%$ & $-29 \%$ & $-7 \%$ & & & \\
\hline \multirow{2}{*}{\multicolumn{11}{|c|}{ AGREGADO MONETARIO M2: relación POSITIVA con la ocurrencia de una crisis }} \\
\hline & & & & & & & & & & \\
\hline $12 \mathrm{~m}$ & $32 \%$ & $24 \%$ & $20 \%$ & $21 \%$ & $19 \%$ & $35 \%$ & $28 \%$ & \multirow{5}{*}{ Argentina } & \multirow{5}{*}{$\begin{array}{c}\text { Brasil, } \\
\text { Venezuela }\end{array}$} & \multirow{5}{*}{$\begin{array}{c}\text { Chile, } \\
\text { Colombia, } \\
\text { México, } \\
\text { Perú }\end{array}$} \\
\hline $9 m$ & $29 \%$ & $41 \%$ & $18 \%$ & $21 \%$ & $17 \%$ & $37 \%$ & $29 \%$ & & & \\
\hline $6 m$ & $27 \%$ & $41 \%$ & $19 \%$ & $19 \%$ & $19 \%$ & $36 \%$ & $65 \%$ & & & \\
\hline $3 m$ & $26 \%$ & $40 \%$ & $19 \%$ & $21 \%$ & $18 \%$ & $36 \%$ & $61 \%$ & & & \\
\hline & $\gamma$ & & 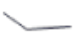 & & 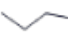 & $\sim$ & - & & & \\
\hline \multicolumn{11}{|c|}{ DEPÓSITOS A LA VISTA: relación NEGATIVA con la ocurrencia de una crisis } \\
\hline $12 \mathrm{~m}$ & $11 \%$ & $1 \%$ & $7 \%$ & $7 \%$ & $2 \%$ & $0 \%$ & $26 \%$ & \multirow{5}{*}{$\begin{array}{l}\text { Argentina, } \\
\text { Brasil, } \\
\text { Chile, Perú }\end{array}$} & \multirow{5}{*}{ Venezuela } & \multirow{5}{*}{$\begin{array}{l}\text { Colombia, } \\
\text { México }\end{array}$} \\
\hline $9 m$ & $11 \%$ & $4 \%$ & $7 \%$ & $8 \%$ & $2 \%$ & $4 \%$ & $22 \%$ & & & \\
\hline $6 m$ & $17 \%$ & $4 \%$ & $10 \%$ & $8 \%$ & $3 \%$ & $4 \%$ & $21 \%$ & & & \\
\hline $3 m$ & $20 \%$ & $3 \%$ & $13 \%$ & $8 \%$ & $1 \%$ & $11 \%$ & $22 \%$ & & & \\
\hline & & & & & -1 & $\longrightarrow$ & $\underbrace{2}$ & & & \\
\hline \multicolumn{11}{|c|}{ TIPO DE INTERÉS: relación POSITIVA con la ocurrencia de una crisis } \\
\hline $12 \mathrm{~m}$ & 6,52 & 3,45 & 1,88 & 1,65 & 2,18 & 0,30 & 4,72 & \multirow{5}{*}{$\begin{array}{l}\text { Chile, } \\
\text { Colombia, } \\
\text { México, } \\
\text { Perú }\end{array}$} & & \\
\hline $9 m$ & 7,14 & 6,93 & 2,09 & 2,27 & 0,44 & 0,30 & 4,09 & & & \\
\hline $6 m$ & 6,51 & 7,63 & 0,75 & 1,43 & 1,80 & 0,29 & 3,50 & & Argentina, & Venezuela \\
\hline $3 m$ & 7,91 & 10,45 & 0,75 & 1,03 & 1,03 & 0,16 & 4,60 & & & \\
\hline & & 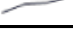 & & & . & & & & & \\
\hline ÍNDIC & E PREC & AL COI & MO: rela & POSITI & on la oc & cia de $u$ & isis & & & \\
\hline $12 m$ & $11 \%$ & $8 \%$ & $5 \%$ & $11 \%$ & $6 \%$ & $6 \%$ & $34 \%$ & & & \\
\hline $9 m$ & $12 \%$ & $9 \%$ & $5 \%$ & $20 \%$ & $6 \%$ & $6 \%$ & $34 \%$ & & & $\begin{array}{l}\text { Argentina, } \\
\text { Chile. }\end{array}$ \\
\hline $6 m$ & $12 \%$ & $18 \%$ & $6 \%$ & $19 \%$ & $6 \%$ & $6 \%$ & $32 \%$ & & Brasil, & México, \\
\hline $3 m$ & $12 \%$ & $291 \%$ & $5 \%$ & $19 \%$ & $6 \%$ & $6 \%$ & $34 \%$ & & & $\begin{array}{c}\text { Perú, } \\
\text { Venezuela }\end{array}$ \\
\hline
\end{tabular}


Tabla 6 (continúa)

Valor de los puntos de corte en cada ventana temporal de predicción

\begin{tabular}{|lccccccccc|}
\hline & ARG & BRA & CHI & COL & MEX & PER & VEN & \multicolumn{2}{c|}{$\begin{array}{c}\text { EVOLUCIÓN DEL PUNTO DE CORTE } \\
\text { - exigente }\end{array}$} \\
+ exigente & Constante \\
\hline ÍNDICE DE PRODUCCIÓN INDUSTRIAL: relación NEGATIVA con la ocurrencia de una crisis & & & \\
$\mathbf{1 2 m}$ & $-7 \%$ & $-6 \%$ & $-2 \%$ & $-11 \%$ & $-8 \%$ & $-14 \%$ & - & & Brasil, \\
$\mathbf{9 m}$ & $-2 \%$ & $-6 \%$ & $-2 \%$ & $-12 \%$ & $-5 \%$ & $-5 \%$ & - & Argentina, & Chile, \\
$\mathbf{6 m}$ & $-3 \%$ & $-6 \%$ & $-2 \%$ & $-10 \%$ & $-3 \%$ & $-4 \%$ & - & México, & Colombia \\
$\mathbf{3 m}$ & $-3 \%$ & $-6 \%$ & $-1 \%$ & $-11 \%$ & $-1 \%$ & $-1 \%$ & - & Perú & \\
\hline
\end{tabular}

Nota: Los cálculos se realizan para las 4 ventanas temporales de predicción incluidas en el análisis: 12 meses (12m); 9 meses $(9 \mathrm{~m}) ; 6$ meses $(6 \mathrm{~m})$ y 3 meses $(3 \mathrm{~m})$. En las tres últimas columnas se incluyen los países en los que la evolución del punto de corte a medida que la ventana temporal se reduce es menos exigente (el punto de corte se corresponde con valores menos atípicos); más exigente (el punto de corte se corresponde con valores más atípicos); o permanece constante.

Fuente: Elaboración propia.

En las últimas columnas de la tabla se identifican los países en los cuales la evolución de los puntos de corte de cada indicador permanece constante (o prácticamente constante) en todas las ventanas temporales o, por el contrario, varía según se aproxima la crisis. En los casos en los que el punto de corte permanece constante en todas las ventanas de predicción, la identificación de un punto de corte móvil no sería necesaria, ya que el valor a partir del cual se emite una señal es el mismo independientemente del momento en que se haga la predicción. Este es el caso del índice de precios al consumo, en el que los puntos de corte se mantuvieron constantes en 5 de los 7 países analizados, o el de la M2 donde no se detectaron variaciones del punto de corte en 4 de los 7 países de la muestra.

Sin embargo, la situación más común es que los puntos de corte no permanezcan constantes, lo que ocurre en al menos algún país de todos los indicadores cuando la ventana temporal se reduce. Merece la pena destacar el caso de las exportaciones, indicador en el que todos los países registraron una variación de los puntos de corte, así como el de las reservas, el tipo de cambio real y el tipo de interés, donde en 6 de los 7 países analizados los umbrales variaron.

La variación que registran los puntos de corte puede tener dos sentidos: el punto de corte se vuelve más exigente (umbrales correspondientes a valores más atípicos) a medida que la ventana temporal se reduce, lo que se asocia a situaciones en las que la crisis termina ocurriendo sólo cuando el indicador evoluciona negativamente en el tiempo; o, el caso contrario, en el que el valor del umbral es cada vez menos exigente (valores de corte cada vez menos atípicos), lo que estaría indicando que aunque el indicador no sigue deteriorándose, o incluso mejora, la crisis ocurre igualmente. Es decir, en el primer caso se encuentran los indicadores que podría deteriorar su evolución a medida que se acerca la crisis (mayor caída en los indicadores que se relacionan negativamente con la crisis; o 
mayor crecimiento en los que lo hacen positivamente), lo cual exigirá ser más exigente con el valor del punto de corte en las ventanas temporales de predicción más próximas a la crisis. En estos casos, mantener el mismo punto de corte que el identificado en la ventana anterior, no tendrá un impacto sobre la predicción de la crisis, ya que si la crisis es predicha con el primer umbral también lo será cuando el nivel de exigencia de éste incremente. Sin embargo, el cálculo de puntos de corte móvil, con un ajuste más preciso a medida que la crisis se acerca, podría reducir el número de falsas señales.

Por ejemplo, en el caso de la variable reservas, cuya relación con la crisis es negativa ya que un menor crecimiento de la variable se asocia con la ocurrencia del shock, el punto de corte en Argentina se vuelve más exigente a medida que la ventana temporal se reduce (pasa de un -19\% en la ventana de 12 meses a un $-35 \%$ en la de 3). En este caso, mantener el punto de corte en $-19 \%$ en todas las ventanas de predicción garantiza que la crisis será predicha en todos los casos ya que, si las reservas caen más del 19\% los 12 meses antes de la crisis y su evolución empeora en el tiempo, su valor seguirá superando el umbral de -19\% los 3 meses antes de que el shock ocurra. Sin embargo, quizá alguna de estas predicciones podría dar lugar a una falsa alarma, en el sentido de que la crisis finalmente no ocurrió. Esto es así ya que, el menor valor del punto de corte para la ventana temporal de 3 meses, indica que para que las crisis terminen ocurriendo la evolución del indicador debe deteriorase significativamente desde los 12 meses hasta los 3 meses antes del shock. Lo mismo ocurre en Brasil con el indicador M2, el cual tiene una relación positiva con la ocurrencia de la crisis, donde el punto de corte aumenta a medida que las ventanas temporales se reducen. Este hecho alerta, nuevamente, de que cuando las crisis terminan ocurriendo este indicador registra en Brasil un fuerte deterioro desde los 12 y hasta los 3 meses antes de la crisis.

Sin embargo, en otros casos ocurre lo contrario, observándose cierta mejora en la evolución del indicador a medida que la crisis está más cerca. Este hecho podría deberse, en parte, a que las medidas de ajuste implementadas por los gobiernos para defender sus monedas tienen un impacto positivo en la evolución del indicador: menor caída en los indicadores que se relacionan negativamente con la crisis, o menor crecimiento en los que lo hacen positivamente. En estos casos, el punto de corte debería ser menos exigente cuando la crisis está cerca para así evitar que ésta no sea predicha. Es decir, si a pesar de la mejora en la evolución del indicador la crisis termina ocurriendo, quizá porque las medidas implementadas por el gobierno no fueron suficientes o no fueron percibidas como suficientes por los agentes del mercado, el nuevo punto de corte deberá ser menos exigente al de la ventana anterior, ya que, aunque el valor del indicador se habrá alejado del umbral inicial, la crisis terminó ocurriendo. Este es el comportamiento que registra, por ejemplo, Brasil en el indicador de exportaciones y Argentina en el de M2. En ambos casos el punto de corte se vuelve menos exigente a medida 
que la ventana temporal se reduce pasando de una caída interanual del -7\% al -1\% en el caso de las exportaciones brasileñas, y de un crecimiento del 32\% al 26\% en la M2 de Argentina. En estos casos, mantener los puntos de corte fijos en todas las ventanas de predicción conduciría a aumentar el número de crisis perdidas. Esto es así ya que, dado que la evolución tiende a mejorar, para el punto de corte inicial los indicadores no emitirán señales cuando la crisis está más cerca.

Según los resultados obtenidos este último comportamiento es el que más se observa en los indicadores, es decir, en mayor medida los indicadores suelen mejorar cuando la crisis se acerca. Esto ocurre sobre todo en el indicador de exportaciones, donde 6 de los 7 países analizados registran puntos de corte menos exigentes a medida que se aproxima la crisis, en el indicador de tipo de cambio donde lo hicieron 5 países, y en los indicadores de depósitos a la vista y tipos de interés donde hubo 4 países que siguieron este patrón. Sin embargo, los casos en los que el punto de corte tiende a ser más exigente según se reduce la ventana temporal son menores. El indicador en el que más países registraron este comportamiento, 3 de los 7 analizados, fue el de las reservas, mientras que en el índice de producción industrial ningún país registró este comportamiento.

\subsection{Resumen de resultados}

En la Tabla 7 se resumen los resultados obtenidos en relación a la heterogeneidad detectada entre países y a la variación de los puntos de corte en cada ventana temporal de predicción. En la parte de arriba de la tabla se encuentran los 5 indicadores en los cuales se identificó una elevada heterogeneidad entre países, lo que aconseja su análisis en muestras separadas para poder identificar percentiles distintos en cada país. Es el caso de las reservas, el tipo de cambio real, exportaciones, depósitos a la vista e índice de producción industrial. Por su parte, la dispersión entre países fue menor para los indicadores de precios, M2 y tipo de interés, aunque en este último caso la dispersión aumentó en las ventanas de predicción más reducidas, por lo que se aconseja también su no inclusión en muestras agregadas de países. En resumen, podríamos decir que para los indicadores relacionados con el sector exterior y con el sector financiero (excepto M2) se aconseja el análisis de muestras individualizadas por país, al menos en el área latinoamericana.

Las celdas sombreadas en la Tabla 7 recogen los casos donde los puntos de corte variaron a medida que la predicción se aproximaba a la llegada de la crisis. El valor de "menos" se asocia a que el punto de corte se vuelve menos exigente según se va reduciendo el horizonte temporal de predicción, mientras que el valor de "más" recoge la situación contraria. Las celdas en las que aparece el símbolo "igual" indican situaciones en las que los puntos de corte permanecieron constantes. Los resultados muestran que casi en el $70 \%$ de los casos analizados se registraron variaciones en los puntos de corte, por lo que se aconseja su análisis 
desagregado para distintas ventanas temporales de predicción. El indicador en el que con mayor frecuencia ocurre esto es el de las exportaciones, seguido de las reservas, el tipo de cambio real, los tipos de interés y los depósitos a la vista. Por países, aquellos en los que se registra mayor variación de los puntos de corte según el horizonte temporal son Argentina, Brasil y Perú, seguidos de Colombia, México y Venezuela. Chile es el único país en el que se registró una evolución más estable, ya que sólo 3 de los 8 indicadores analizados registraron variaciones.

Tabla 7

Identificación de puntos de corte "móviles" en la muestra analizada

\begin{tabular}{|c|c|c|c|c|c|c|c|c|c|c|c|}
\hline & \multirow[b]{2}{*}{ ARG } & \multirow[b]{2}{*}{ BRA } & \multirow{2}{*}{\multicolumn{2}{|c|}{$\mathrm{CHI}$ COL }} & \multirow[b]{2}{*}{ MEX } & \multirow{2}{*}{\multicolumn{2}{|c|}{ PER VEN }} & \multicolumn{4}{|c|}{$\begin{array}{l}\text { EVOLUCIÓN DEL PUNTO DE CORTE } \\
\text { (número de casos) }\end{array}$} \\
\hline & & & & & & & & - exigente & + exigente & $\begin{array}{l}\text { Puntos } \\
\text { de corte } \\
\text { MÓVIL }\end{array}$ & $\begin{array}{c}\text { Puntos de } \\
\text { corte } \\
\text { CONSTANTE }\end{array}$ \\
\hline \multicolumn{12}{|c|}{ ELEVADA HETEROGENEIDAD ENTRE PAÍSES } \\
\hline Reservas & + & - & $=$ & - & + & + & - & 3 & 3 & 6 & 1 \\
\hline Tipo de cambio real & - & - & $=$ & - & - & - & + & 5 & 1 & 6 & 1 \\
\hline Exportaciones & + & - & - & - & - & - & - & 6 & 1 & 7 & 0 \\
\hline Depósitos a la vista & - & - & - & $=$ & $=$ & - & + & 4 & 1 & 5 & 2 \\
\hline Índice de producción industrial & - & $=$ & $=$ & $=$ & - & - & & 3 & 0 & 3 & 3 \\
\hline \multicolumn{12}{|c|}{ BAJA HETEROGENEIDAD ENTRE PAÍSES } \\
\hline Tipo de interés & + & + & - & - & - & - & $=$ & 4 & 2 & 6 & 1 \\
\hline Índice de precios al consumo & $=$ & + & $=$ & + & $=$ & $=$ & $=$ & 0 & 2 & 2 & 5 \\
\hline Agregado monetario M2 & - & + & $=$ & $=$ & $=$ & $=$ & + & 1 & 2 & 3 & 4 \\
\hline \multicolumn{12}{|c|}{ EVOLUCIÓN DEL PUNTO DE CORTE (número de casos) } \\
\hline - exigente & 4 & 4 & 3 & 4 & 4 & 5 & 2 & $26(47 \%)$ & & & \\
\hline + exigente & 3 & 3 & 0 & 1 & 1 & 1 & 3 & & $12(22 \%)$ & & \\
\hline Puntos de corte MóVIL & 7 & 7 & 3 & 5 & 5 & 6 & 5 & & & 38 (69\%) & \\
\hline Puntos de corte CONSTANTE & 1 & 1 & 5 & 3 & 3 & 2 & 2 & & & & $17(31 \%)$ \\
\hline
\end{tabular}

Nota: Las celdas sombreadas representan situaciones donde el punto de corte varió a medida que lo hizo la ventana temporal de predicción. El signo "+" indica que el punto de corte tiende a ser más exigente a medida que se reduce el horizonte temporal; el singo "-" indica que el punto de corte tiende a ser menos exigente a medida que se reduce la ventana temporal; el signo "=" indica que los puntos de corte no variaron en las distintas ventanas temporales analizadas.

Fuente: Elaboración propia.

En relación al signo de las variaciones registradas en los puntos de corte, del total de casos que registraron una variación en casi el 70\% se produjo una reducción en el nivel de exigencia de los puntos de corte (umbrales correspondientes a valores menos atípicos) a medida que la crisis se aproxima. Estas situaciones podrían estar asociadas al hecho de que, la aplicación de medidas por parte del gobierno para frenar la llegada de la crisis puede suponer una "falsa" mejora en la evolución de estos indicadores, cuyos niveles aparentemente mejorados, sin embargo, terminarán conduciendo a una crisis si las políticas de ajuste implementadas no resultaron suficientes. En los indicadores en los que se registraron variaciones en el punto de corte, este es el comportamiento que ocurre con mayor frecuencia. Tan sólo en el caso de las 
reservas la situación no es tan clara, ya que el número de países en los que el nivel de exigencia se reduce es el mismo en los que aumenta. Por países, es en Colombia, México y Perú donde prácticamente todos los indicadores en los que el punto de corte varió lo hizo reduciendo su nivel de exigencia. En Chile, los únicos tres indicadores en los que se registró variación del punto de corte también lo hicieron en este sentido. Por su parte, en Argentina, Brasil y Venezuela, los resultados son mixtos.

En resumen, los resultados obtenidos muestran que tan sólo dos de los indicadores analizados (índice de precios al consumo y M2) registran un comportamiento más o menos estable tanto en su comparativa entre países como cuando se analiza su evolución temporal según se aproxima la crisis. Sin embargo, en el resto de indicadores su capacidad predictiva podría mejorarse si los análisis se hicieran de manera más minuciosa, teniendo en cuenta muestras aisladas de países e identificando ventanas temporales de predicción que mostrasen un mayor nivel de desagregación a medida que la crisis se aproxima.

\section{CONCLUSIONES}

Si bien la diversidad y heterogeneidad que caracteriza tanto a las crisis como a los países que las sufren reduce la capacidad predictiva de los Sistemas de Alerta Temprana, las restricciones que impone la construcción de modelos econométricos, en los que la muestra debe ser elevada para garantizar la consistencia de los resultados, obliga a trabajar con muestras multi-país en la mayoría de los casos. Sin embargo, el empleo de técnicas no paramétricas, a través del enfoque de señales, facilita el análisis a nivel país, lo que podría evitar el impacto que la heterogeneidad tiene sobre la capacidad predictiva de los Sistemas de Alerta Temprana. Este trabajo pretende realizar un ejercicio pormenorizado para ver si esa heterogeneidad está presente en las crisis ocurridas en siete países latinoamericanos. Además, en el trabajo también se analiza si los puntos de corte, a partir de los cuales el indicador emite una señal de que una crisis va a ocurrir, deberían variar según el horizonte temporal de predicción. Con ello se pretende prestar atención a uno de los objetivos de los gobiernos, quienes querrán priorizar la reducción del número de crisis perdidas o no predichas por los Sistemas de Alerta, y no sólo reducir el número de falsas alarmas emitidas. En este sentido, si los puntos de corte fueran capaces de ajustarse a la evolución que los indicadores siguen a medida que la crisis se aproxima, el número de crisis no predichas podría verse reducido.

Los resultados obtenidos en este trabajo confirman la existencia de heterogeneidad entre países, por lo que si el percentil, que permite identificar el punto de corte o umbral a partir del cual el indicador emite una señal, es calculado de manera individual para cada país la capacidad predictiva de los Sistemas de Alerta Temprana podría verse mejorada. En concreto, estos resultados afectan 
sobre todo a los indicadores relacionados con el sector exterior y con el sector financiero.

Además, los resultados aportan también evidencia sobre la conveniencia de identificar puntos de corte móviles que varíen según la crisis se aproxima. En la mayoría de los casos estos puntos de corte se vuelven menos exigentes a medida que se reduce el horizonte temporal de predicción, quizá debido a que la aplicación de medidas por parte de los gobiernos, una vez que ha sido identificada la crisis, influye positivamente en la evolución de los indicadores. Con ello, la mejora registrada por los indicadores puede aparentar una falsa sensación de que la crisis ya no ocurrirá. Es decir, ésta será una crisis perdida o no identificada por el Sistema de Alerta en la medida en que el nivel del indicador no superará el punto de corte identificado en la ventana temporal más alejada de la crisis. Sin embargo, si las medidas aplicadas no son suficientes, la crisis terminará ocurriendo a pesar de la mejora registrada por el indicador. Para evitar que el Sistema de Alerta falle en la anticipación de estas crisis es necesario ser menos exigente en la definición del punto de corte a medida que la ventana temporal de predicción se acerca a la llegada de la crisis, lo cual se consigue con la identificación de los puntos de corte móviles.

En concreto, los indicadores en los que los puntos de corte mostraron más variación son: exportaciones, tipo de interés, tipo de cambio, reservas internacionales y depósitos a la vista. En todos estos indicadores, aunque la evolución de los puntos de corte identificados registró mejoría a medida que las ventanas temporales de predicción se reducían, la crisis finalmente ocurrió. En el caso de las reservas, la evolución de los puntos de corte en determinados países también registró el comportamiento contrario, volviéndose su valor más exigente a medida que se aproximaba la crisis. Esta situación se corresponde con crisis que finalmente ocurren cuando el deterioro del indicador es muy significativo a medida que el shock se acerca.

Con este diseño del enfoque de señales se podría mejorar la capacidad predictiva de los Sistemas de Alerta Temprana al permitir que el umbral definido para que el indicador emita una señal pueda variar en el tiempo. El uso de puntos de corte móviles, frente al empleo de umbrales que no varían durante la ventana temporal de predicción, debería ajustarse mejor a la evolución que registran las condiciones económicas del país a medida que la crisis cambiaria está más cerca en el tiempo, reduciendo con ello el número de crisis perdidas o no predichas por los Sistemas de Alerta. Si bien los resultados de este trabajo se muestran como un ejercicio que sirva para valorar la conveniencia de trabajar con muestras por países, frente a muestras multi-pais, y puntos de corte móviles, frente a puntos de corte estáticos, este mismo diseño del Sistema de Alerta Temprana podría ser generalizado a otros indicadores y/o áreas geográficas para ver si los resultados se repiten. 


\section{REFERENCIAS BIBLIOGRÁFICAS}

ANGKINAND A., LI J., WILLETT T. (2006). "Measures of currency crises: A survey". Claremont Working Papers, 2006-06. Claremont Graduate University: Claremont, CA.

BABECKÝ J., HAVRÁNEK T., MATĚJU゚ J., RUSNÁK M., VAŠíČEK B. (2014). "Banking, debt, and currency crises in developed countries: Stylized facts and early warning indicators". Journal of Financial Stability, 15, pp. 1-17.

BHATTACHARYA B., ROY T.N.S. (2012). "Indicators of Banking Fragility in India: An Empirical Test". South Asia Economic Journal, 13(2), pp. 265-290.

BARBERÁ RA., DONCEL LM., SAINZ J. (2006). “¿Es posible predecir el comportamiento del tipo de cambio? Una aplicación del modelo de Lucas al caso español". Estudios de Economía Aplicada, 24(1), pp. 427-452.

BERG, A., BORENSZTEIN, E., MILESI-FERRETTI, GM., PATTILLO, C. (1999). "Anticipating Balance of Payments Crises: the Role of Early Warning Systems". IMF Occasional Papers, 186, IMF, Washington.

BERG A., PATTILLO C. (1999a). "Are currency crises predictable? A test". IMF Staff Papers, 46 (2), pp. 107-138.

BERG A., PATTILLO C. (1999b). "Predicting currency crises: the indicators approach and an alternative". Journal of International Money and Finance, 18(4), pp. 561-586.

BERG A., PATTILLO C. (1999c). "What caused the Asian crises: An early warning system approach". Economic Notes, 28 (3), pp. 285-334.

BUSSIÈRE M., FRATZSCHER M. (2006). "Towards a new early warning system of financial crises". Journal of International Money and Finance, 25(6), pp. 953-973.

BUSSIÈRE M., MULDER C. (1999). "External Vulnerability in Emerging Market Economies: How high liquidity can offset weak fundamentals and the effects of contagion", IMF Staff Papers, 88.

CAGGIANO G., CALICE P., LEONIDA L., KAPETANIOS G. (2016). "Comparing logitbased early warning systems: Does the duration of systemic banking crises matter?" Journal of Empirical Finance, 37, pp. 104-116.

CANDELON B., DUMITRESCU El., HURLIN C. (2014). "Currency crisis Early Warning Systems: Why they should be dynamic". International Journal of Forecasting, 30(4), pp. 1016-1029.

CARAMAZZA F., RICCI L., SALGADO R. (2004). "International financial contagion in currency crises". Journal of International Money and Finance, 23(1), pp. 51-70.

DAWOOD M., HORSEWOOD N., STROBEL F. (2017). "Predicting sovereing debt crises: An Early Warning System Approach". Journal of Financial Stability, 28, pp. 16-28.

EDISON HJ. (2000). "Do indicators of financial crises work? An evaluation of an Early Warning System". International Finance Discussion Papers, 675.

EICHENGREEN B., ROSE A., WYPLOSZ C. (1994). "Speculative attacks on pegged exchange rates: An empirical exploration with special reference to the European monetary system". NBER Working Paper, 4898.

EICHENGREEN B., ROSE A., WYPLOSZ C. (1995). "Exchange market mayhem: The antecedents and aftermath of speculative attacks". Economic Policy, 21, pp. 249-312.

EICHENGREEN B., ROSE A., WYPLOSZ C. (1996). "Contagious Currency Crisis". NBER Working Paper, 5681. 
EL-SHAZLY, A. (2011). "Designing an early warning system for currency crises: An empirical treatment". Applied Economics, 43(14), pp. 1817-1828.

EMIN D., AYTAC A. (2016). "The challenge of predicting currency crises: how do definition and probability threshold choice make a difference?" Eurasian Economic Review, 6(2), pp. 95-213.

FRANKEL JA., ROSE AK. (1996). "Currency crashes in emerging markets: An empirical treatment". Journal of International Economics, 41, pp. 351-366.

GIRTON L., ROPER D. (1977). "A monetary model of exchange market pressure applied to postwar Canadian experience". American Economic Review, 67(4), pp. 537-548.

HAILE F., POZO S. (2008). "Currency crisis contagion and the identification of transmission channels" International Review of Economics \& Finance, 17(4), pp. 572588.

HEUN M., SCHLINK T. (2004). "Early warning systems of financial crises - Implementation of a currency crisis model for Uganda" HfB - Business School of Finance \& Management, 59.

JACOBS JPAM., KUPER GH., LESTANO L. (2005). "Currency crises in Asia: A multivariate logit approach". CCSO Working Papers 2005/06, University of Groningen.

KAMINSKY G. (1999). "Currency and Banking Crises: The Early Warnings of Distress" IMF Working Paper, 178.

KAMINSKY G., LIZONDO S., REINHART CM. (1998). "Leading Indicators of currency crisis". IMF Staff Papers, 45(1), pp. 1-43.

MEDINA E., VICÉNS J. (2006). "Selección de indicadores adelantados de crisis cambiarias en Latinoamérica bajo un enfoque econométrico". Cuadernos de Economía: Spanhish Journal of Economics and Finance, 29 , pp. 85-118.

PANKKI S. (1999). "Measuring Exchange Market Pressure and Central Bank Intervention". Working Paper, Bank of Finland.

REDONDO D., RODRÍGUEZ JM. (2014). "Crisis en las entidades de crédito españolas: un estudio mediante análisis discriminante" Estudios de Economía Aplicada, 32(2), pp. 617-644.

SACHS J.D., TORNELL A., VELASCO A. (1996). "Financial crises in emerging markets: the lessons from 1995 (with comments and discussion)". Brookings Papers on Economic Activity, 1, pp. 147-198.

ROSE A., SPIEGEL M. (2011). "Cross-country causes and consequences of the crisis: an update". European Economic Review, 55, pp. 309-324.

SEVIM C., OZTEKIN A., BALI O., GUMUS S., GURESEN E. (2014). "Developing an early warning system to predict currency crises". European Journal of Operational Research, 237, pp. 1095-1104.

TANNER E. (2001). "Exchange Market Pressure and Monetary Policy: Asia and Latin America in the 1990s". IMF Staff Papers, 47, pp. 311-333

TORNELL A. (1998). "Common Fundamentals in the Tequila and Asian Crises". NBER Working Paper, 7139.

VORTELINOS DI. (2016). "Evaluation of the Federal Reserve's financial-crisis timeline". International Review of Financial Analysis, 45, pp. 350-355.

ZHANG Z. (2001). "Speculative attacks in the Asian crisis". IMF Working Paper, 189. 
